\title{
Impact of time delays and environmental noise on the extinction of a population dynamics model
}

\author{
Chun Zhang ${ }^{1}$, Tao Yang $^{2, a}{ }_{(\mathbb{D}}$, and Shi-Xian $\mathrm{Qu}^{1}$ \\ 1 School of Physics and Information Technology, Shaanxi Normal University, Xi'an 710062, People's Republic of China \\ 2 Department of Engineering Mechanics, Northwestern Polytechnical University, Xi'an 710072, People's Republic of China
}

Received 29 June 2021 / Accepted 27 September 2021 / Published online 3 November 2021

(C) The Author(s), under exclusive licence to EDP Sciences, SIF and Springer-Verlag GmbH Germany, part of Springer Nature 2021

\begin{abstract}
In this paper, we examine a population model with carrying capacity, time delay, and sources of additive and multiplicative environmental noise. We find that time delay, noise sources and their correlation induce regime shifts and transitions between the population survival state and the extinction state. To explore the transition mechanism between these two states, we analyzed the shift time to extinction, or the delayed extinction time, of populations. The main finding is that the extinction transition time as a function of the noise intensity shows a maximum, indicating the existence of an appropriate noise intensity leading to a maximal delayed extinction. This nonmonotonic behavior, with a maximum, is a signature of the noise-enhanced stability phenomenon, observed in many physical and complex metastable systems. In particular, this maximum increases (or decreases) as the cross-correlation intensity or the delay time in the death process increases. Furthermore, the signal-to-noise ratio as a function of noise intensity shows a maximum, which is a signature of the stochastic resonance phenomenon in the population dynamics model investigated in the presence of time delay and environmental noise.
\end{abstract}

\section{Introduction}

Recently, a large body of research has focused on the cooperative interplay of noise and nonlinearity in dynamical systems, leading to phenomena such as regime shifts $[1,2]$. Regime shifts are substantial, longlasting reorganizations of complex systems. In nonlinear stochastic systems, even a weak noise can generate unexpected phenomena that have no analogues in the deterministic case $[3,4]$. These noise-induced phenomena such as a stochastic resonance $[5,6]$, noiseinduced transitions $[7,8]$, noise-enhanced stability [913], stochastic bifurcations $[14,15]$, noise-induced chaos and order [16-18], and stochastic excitability [19,20] still attract attention of many researchers from the different domains of science [21].

Actually, many interesting stochastic phenomena are observed also in life science $[22,23]$. The effect of noise on the spatial-temporal behavior of the maximum chlorophyll in the deep Mediterranean [24]. They predict the behavior of Listeria monocytogenes under the influence of noise during the fermentation of traditional Sicilian salami [25]. In addition, noise has also played an active and constructive role in other interdisciplinary fields such as materials science, and other stochastic phenomena have appeared. Such as the resonant activation in polymer translocation [26], field- and irradiation-induced phenomena in memristive nanoma-

\footnotetext{
a e-mail: yangtscn@nwpu.edu.cn (corresponding author)
}

terials [27], a selectively laser-melted stainless steel [28] and out-of-equilibrium quantum critical phenomena $[29]$.

On the other side, these investigations on the dynamic properties of the population dynamics may neglect the possible effects induced by time delay. The important effects of time delays in dynamical systems have been brought to light in Refs. [30,31]. In physics, time delays reflect the transmission times related to the transport of matter, energy and information through the system [32]. Furthermore, time delay changes the dynamic properties of the system and brings a series of interesting and significant results [33], for example, time delay induced traveling wave solutions [34], coherence resonance [35], excitability [36], periodically oscillate synchronously [37] and stochastic resonance [38]. Time delay is also very important for issues that affect the survival of humans around the world. For example, the time delay is also a key factor in studying the dynamic mechanism of COVID-19 transmission $[39,40]$. Actually, the effects of time delay and stochasticity on dynamic systems are not always positive [41-43]. However, it appears that the combination of noise and time delay is ubiquitous in nature and often changes fundamentally the dynamics of the systems investigated [4446]. Bistable systems with noise and time delay have been investigated in detail in Refs. [35,47].

Stochastic resonance (SR) is a noise-induced effect demonstrating the phenomenon of signal amplification, which has been extensively investigated in biological 
Table 1 Transition processes and associated rates in population model

\begin{tabular}{ll}
\hline Transition processes & Associated rates \\
\hline$N \rightarrow \varnothing$ & $\mu N$ \\
$2 N \rightarrow 3 N$ & $\lambda N(N-1) /(2 K)$ \\
$3 N \rightarrow 2 N$ & $\sigma N(N-1)(N-2) /\left(6 K^{2}\right)$ \\
\hline
\end{tabular}

systems [48-50]. In particular, such as stochastic resonance and noise-delayed extinction in a model of two competing species [51]; role of the noise on the transient dynamics of an ecosystem of interacting species [52]; and Cyclic fluctuations, climatic changes and role of noise in Planktonic Foraminifera in the Mediterranean Sea [53]. The concept of SR was originally proposed to explain periodic recurrences of the earth's ice age [54], it has attracted researchers' interests, and it has been studied in geophysical, biological, chemical systems, and other fields [55] to manifest the constructive role of noises. Noise-induced resonance phenomena include doubly stochastic resonance [56], SR on bone loss [57], SR in excitable systems [58], coherence resonance [19] and array-enhanced coherence resonance [59]. In this paper, regime shifts between the population survival state and the extinction state induced by the noise sources, their cross-correlation, and by time delays have been observed and investigated. Moreover, the STE as a function of the noise intensity has a nonmonotonic behavior with a maximum, indicating the existence of an appropriate noise intensity leading to a maximal delayed extinction. This nonmonotonic behavior is a signature of the noise-enhanced stability phenomenon (NES) observed in many physical and complex metastable systems. Finally, the stochastic resonance (SR) phenomenon of the population model with time delay and noises is observed and investigated. The paper is organized as follows. In Sect. 2, the population model subject to noises and time delay is presented, and then the impacts of the noises and time delay on the time series, probability distribution, STE, NES and SR are discussed in Sect. 3, respectively. Finally, conclusions are given in Sect. 4.

\section{Description of population models}

\subsection{Deterministic description of population model}

Consider a specific example first where the local dynamics displays the Allee effect [60]; i.e., in a certain range of parameters the single patch dynamics is bistable: there is one stable state with population survival state and another corresponding to the extinction state. The corresponding population model is represented by the transition processes and associated rates in Table 1.

The first two transitions are required to capture the Allee effect. The death rate of a low-density population is given by $\mu$, and the birth rate of the population when

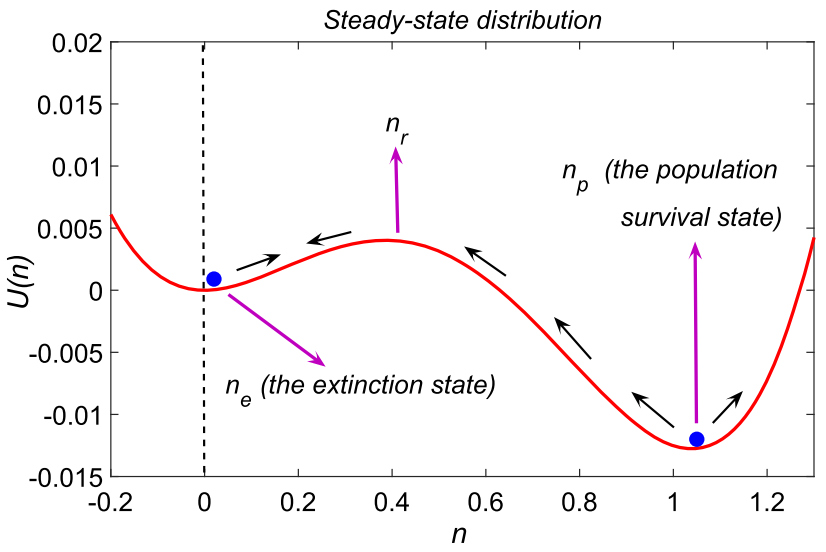

Fig. 1 Deterministic description of population model. The potential $U(n)$ as a function of $n$ with $\mu=0.2, \sigma=3.0$, $\lambda=1.425$. For different initial conditions, the $n(t)$ can be distributed at one of the two steady states $\left(n_{e}\right.$ and $\left.n_{p}\right)$

the density is large enough is given by $\lambda$. The negative birth rate for an overcrowded population is provided by $\sigma$, and $K$ is the carrying capacity of the population. Therefore, the deterministic rate equation has the form [61]:

$$
\frac{\mathrm{d} n(t)}{\mathrm{d} t}=\underbrace{-\frac{\sigma}{6} n^{3}(t)+\frac{\lambda}{2} n^{2}(t)}_{\text {birth }} \underbrace{-\mu n(t)}_{\text {death }}=h(n(t))
$$

When $\Delta^{2}=1-8 \sigma \mu /\left(3 \lambda^{2}\right)>0$, this equation has three fixed points and, therefore, describes a significant Allee effect. The fixed points $n_{e}=0$ (the extinction state) and $n_{p}=K(1+\Delta)$ (the population survival state) are attracting, the fixed point $n_{r}=K(1-\Delta)$ is repelling. The fixed point $n_{r}$ corresponds to the critical population size for establishment, whereas $n_{p}$ corresponds to the established population. The parameter $K=3 \lambda /(2 \sigma)$ sets the scale of the established population size. The potential function corresponding to Eq. (1) is

$$
\begin{aligned}
U(n(t)) & \left.=-\int^{n(t)} h(n(t))\right) \mathrm{d} n(t) \\
& =\frac{\sigma}{24} n^{4}(t)-\frac{\lambda}{6} n^{3}(t)+\frac{\mu}{2} n^{2}(t)
\end{aligned}
$$

An interesting aspect of the model is that, based on the different initial conditions, $n(t)$ can be distributed at one of the two stable steady states $\left(n_{e}\right.$ and $\left.n_{p}\right)$. It is a bistable system for certain values of $\mu, \sigma, \lambda$. Bistability is a kind of important dynamical feature in this system, especially for the fate decision in some processes. In this article, our works are employed in the bistable region as shown in Fig. 1. It pointed out that dynamics of the population size according to the mean-field theory corresponds to the coordinate $n(t)$ of an overdamped particle, performing deterministic motion in this potential. 


\subsection{Stochastic description of population model with time delay}

Population systems are often subject to environmental noise $[62,63]$. It is therefore useful to reveal how the noise affects the delayed population systems. It has been well known in physics, biology, complexity science, control theory, and econophysics $[9,64,65]$ that noise can also have a stabilizing effect [10-13]. It has also been revealed recently by Mao, Marion, and Renshaw [66] that the environmental noise can suppress a potential population explosion. These indicate clearly that different structures of environmental noise may have different effects on the population systems. Therefore, it is reasonable to study the effects of random fluctuations on the population model is given by

$$
\frac{\mathrm{d} n(t)}{\mathrm{d} t}=h(n(t))+\sum_{j=1}^{2} g_{j}(n(t)) \xi_{j}(t)
$$

where $g_{j}(n(t))$ are deterministic functions that characterize the state dependent action of Gaussian noises $\xi_{j}(t)$, and the noises are white with zero mean which obeys $\left\langle\xi_{j}(t) \xi_{j}\left(t^{\prime}\right)\right\rangle=2 d_{j} \delta\left(t-t^{\prime}\right), d_{j}$ are the intensities of the noises $\xi_{j}(t)$, respectively. In our model, we simultaneously consider both the multiplicative $\left[g_{1}(n(t))=\right.$ $-n(t)]$ and additive $\left[g_{2}(n(t))=1\right]$ noises. The environmental fluctuations act also in the death rate constant $\mu$, so the control parameter $\mu$ is replaced by $\mu+\xi_{1}(t)$, and as additive noise source $\xi_{2}(t)$. The additive noise describes the inherent uncertainties due to the existence of alternative attractors. The two independent noises $\xi_{1}(t)$ and $\xi_{2}(t)$ may have a common source, thereby the correlation between them should be taken into account in our model, such that $\left\langle\xi_{1}(t) \xi_{2}\left(t^{\prime}\right)\right\rangle=\left\langle\xi_{2}(t) \xi_{1}\left(t^{\prime}\right)\right\rangle=$ $2 q \sqrt{d_{1} d_{2}}$, where $q$ is the intensity characterizing the cross-correlation of the noises, $|q| \leq 1$.

On the other hand, all processes take time to complete. While physical processes such as acceleration and deceleration take little time compared to the times needed to travel most distances. However, the times involved in biological processes such as gestation and maturation can be substantial when compared to the data-collection times in most population studies. Therefore, it is often imperative to explicitly incorporate these process times into mathematical models of population dynamics. These process times are often called delay times, and the models that incorporate such delay times are referred as delay differential equation (DDE) models. The stochastic delay Langevin equation corresponding to this population model is given by

$$
\frac{\mathrm{d} n(t)}{\mathrm{d} t}=h\left(n(t), n\left(t-\tau_{i}\right)\right)+\sum_{j=1}^{2} g_{j}\left(n(t), n\left(t-\tau_{i}\right)\right) \xi_{j}(t)
$$

The time delays $\tau_{i}$ could appear at any level of population model, like in the death, birth or global process.
Table 2 Note that $n(t)=n, n\left(t-\tau_{i}\right)=n_{\tau_{i}}$

\begin{tabular}{ll}
\hline Time delay & Equation: $h\left(n, n_{\tau_{i}}\right)$ \\
\hline$\tau_{d}$ in the death process & $-\frac{\sigma}{6} n^{3}+\frac{\lambda}{2} n^{2}-\mu n_{\tau_{d}}$ \\
$\tau_{b}$ in the birth process & $-\frac{\sigma}{6} n_{\tau_{b}}^{3}+\frac{\lambda}{2} n_{\tau_{b}}^{2}-\mu n$ \\
$\tau_{g}$ in the global process & $-\frac{\sigma}{6} n_{\tau_{g}}^{3}+\frac{\lambda}{2} n_{\tau_{g}}^{2}-\mu n_{\tau_{g}}$ \\
\hline
\end{tabular}

We estimate the effects induced by time delay $\tau_{d}$ in the death process, $\tau_{b}$ in the birth process and $\tau_{g}$ in the global process.

The modified model I: we first include the local time delay $\tau_{d}$ in the death process, i.e., the death term $-\mu n$ can be written as $-\mu n_{\tau_{d}}$. According to Table 2, the stochastic delay differential equation (4) is further rewritten:

$$
\frac{\mathrm{d} n}{\mathrm{~d} t}=-\frac{\sigma}{6} n^{3}+\frac{\lambda}{2} n^{2}-\left(\mu+\xi_{1}(t)\right) n_{\tau_{d}}+\xi_{2}(t)
$$

where the $\tau_{d}$ previous to the time when $\mathrm{d} n / \mathrm{d} t$ is computed. Since $\mu n_{\tau_{d}}$ is dependent linearly on the population, for simplicity, we call this form of time delay as linear time delay. In addition, only small time delay is investigated in the modified model I, since the theoretical approximation methods below are applicable for the small delay time.

The modified model II: since time delays in population models often account for maturation or gestation periods, we include the local time delay into the birth term (the rates constant $\sigma$ and $\lambda$ ):

$$
\frac{\mathrm{d} n}{\mathrm{~d} t}=-\frac{\sigma}{6} n_{\tau_{b}}^{3}+\frac{\lambda}{2} n_{\tau_{b}}^{2}-\left(\mu+\xi_{1}(t)\right) n+\xi_{2}(t)
$$

where the first and second terms on the right side is evaluated at a time $\tau_{b}$ previous to the time when $\mathrm{d} n / d t$ is computed, and is nonlinear time-delayed, and the delay time does not appear in the stochastic force. For simplicity, we regard this case as nonlinear time delay case.

The modified model III: lastly, we consider the inclusion of both the delay appearing in death and birth processes:

$$
\frac{\mathrm{d} n}{\mathrm{~d} t}=-\frac{\sigma}{6} n_{\tau_{g}}^{3}+\frac{\lambda}{2} n_{\tau_{g}}^{2}-\left(\mu+\xi_{1}(t)\right) n_{\tau_{g}}+\xi_{2}(t)
$$

In this equation, $\tau_{g}$ is a global delay in the population model, this case combines the impacts of the above two cases. The statistics properties of our theoretical model subjected to correlated noises and time delays are explored in different cases.

\section{Main results and discussion}

To investigate the roles of time delays and environmental noise on catastrophic regime shifts in popula- 
tion model, we study numerically the time series, the probability density and mean first shift time of the population. The numerical simulations are performed by directly integrating the stochastic delay differential equation (4). The Box-Mueller algorithm is used to generate Gaussian noise [67]. The numerical data of probability distribution are obtained using the Euler procedure with a time step of $h=0.01$, and the data of probability distribution are saved over 500 different trajectories. We performed 100,000 simulations to determine this limit of stability.

\subsection{Time series and probability density of population}

In this subsection, we estimate the impacts induced by noises and delays on the probability density to analyze the regime shift phenomenon. Let $P(n, t)$ denotes the probability density distribution that the probability exactly equals $n$ at time $t$. Then, the delay FokkerPlanck equation of $P(n, t)$ corresponding to Eq. (4) can be given by [44]

$$
\begin{aligned}
\frac{\partial P(n, t)}{\partial t}= & -\frac{\partial}{\partial n} F\left(\left(n, n_{\tau_{i}}\right)\right) P(n, t) \\
& +\frac{\partial^{2}}{\partial n^{2}} G\left(\left(n, n_{\tau_{i}}\right)\right) P(n, t)
\end{aligned}
$$

where

$$
\begin{aligned}
& F\left(n, n_{\tau_{i}}\right)=h\left(n, n_{\tau_{i}}\right)+\frac{1}{2} G^{\prime}\left(n, n_{\tau_{i}}\right) \\
& G\left(n, n_{\tau_{i}}\right)=d_{1} g_{1}^{2}\left(n, n_{\tau_{i}}\right)+2 q \sqrt{d_{1} d_{2}} g_{1}\left(n, n_{\tau_{i}}\right)+d_{2}
\end{aligned}
$$

In Figs. 2 and 3, the impacts of the intrinsic noise $d_{1}$ and extrinsic noise intensity $d_{2}$ on the probability density of population are plotted by directly simulating the Langevin equation (4). As the value of $d_{1}$ increases (see $d_{1}=0.01, d_{1}=0.05$, and $d_{1}=0.20$ in Fig. 2 ), the peak of $n_{e}$ state becomes higher, and the $n_{p}$ becomes lower. The above result indicates that the population model is affected by the internal noise $d_{1}$ and will switch from the population survival state $n_{p}$ to the extinct state $n_{e}$. However, as the extrinsic noise $d_{2}$ increase $\left(d_{2}=0.003\right.$, $d_{2}=0.005$, and $\left.d_{2}=0.020\right)$, the structure of the probability density of population changes from $n_{e}$ state to $n_{p}$ state, as shown in Fig. 3. Therefore, the two noise intensities have different effects on the population system. To get more physical insight into the noise-induced transition phenomenon investigated, we cite an example to illustrate. We have fixed parameters are the same as in Fig. $3\left(d_{2}=0.005\right)$. In Fig. 8 , we have calculated the PDF at different times $(t=0, t=10, t=100$, and $t=1000)$, corresponding to the stationary state. And we can see the time evolution of the PDF from a peaked delta-function at $t=0$ towards the stationary double peak. Figure 4 shows the influence of different cross-correlation intensity $q$ on probability density of population. For the negative cross-correlation intensity $(q=-0.9)$, the probability density as a function of $n$ shows the extinct state $n_{e}$ state. As the value of crosscorrelation intensities increases $(q=0.1)$, the structure of the probability density of population exhibits two peaks. When the $q=0.9$, the transition from two peaks to single peaks. In other words, as the cross-correlation intensity increases, the probability density of population changes from extinct state $n_{e}$ to population survival state $n_{p}$. We can understand that if the population system is to remain extinct state $n_{e}$, then the cross-correlation intensity need to reduce.

Figures 5, 6 and 7 depict that probability density of population $n$ for different time delays $\tau_{d}, \tau_{b}$ and $\tau_{g}$, respectively. When time delays $\tau_{d}=\tau_{b}=\tau_{g}=0.1$, the probability density of population as a function of $n$ exhibits two peaks, one is at $n_{e}$ state, and the other is at $n_{p}$ state. As the value of degradation delay $\tau_{d}$ increases, the structure of the probability distribution switches from population survival state $n_{p}$ to extinct state $n_{e}$ in the population system. However, the case of $\tau_{b}=1.0$ (Fig. 6) is different from $\tau_{d}$ (Fig. 5) and $\tau_{g}$ (Fig. 7) increase. With the increase of $\tau_{b}=2.0$, the phenomenon of two peaks becomes more obvious. In other words, the population survival state $n_{p}$ and extinction state $n_{e}$ coexist.

\subsection{The shift time to extinction and NES}

The system possesses two stable states: one is the population survival state $\left(n_{p}\right)$, and another is the extinction state $\left(n_{e}\right)$. Environmental perturbations and time delays present in the population system can induce regime shifts between the two alternative stable states. A quantity of interest is the time from the population survival state to the extinction state. This time is a random variable and is often referred to as the first shift time. It is necessary for us to study the mean first shift time from the population survival state to the extinction state. A simple but very general approach to the classical mean first shift time problem was given in Refs. [68], which has been extended by us for investigation the shift time to extinction (STE) of populations. We assume that all populations in the simulations are initial located at position $n(t=0)=n_{p}$ (the population survival state). The $\operatorname{STE} T(n ; a, b)$ is the average time of the first exit from the interval $(a, b)$ and satisfied the question [69]:

$$
-1=F\left(n, n_{\tau_{i}}\right) \frac{\mathrm{d} T(n)}{\mathrm{d} n}+\frac{1}{2} G\left(n, n_{\tau_{i}}\right) \frac{d^{2} T(n)}{\mathrm{d} n^{2}}
$$

where the drift and diffusion coefficients $F\left(n, n_{\tau_{i}}\right)$ and $G\left(n, n_{\tau_{i}}\right)$ are given in equation (9) and (10), respectively. The STE $T(n)=T\left(n_{p} \rightarrow n_{e}\right)$ for exit from the basin of attraction of the stable steady state at $n_{e}$ (the extinction state) is obtained with the interval $(a, b)=\left(0, n_{p}\right)$ and boundary conditions given by $T^{\prime}(a ; a, b)=0$ and $T(b ; a, b)=0$. The prime denotes differentiation with respecting to $n$, with reflecting and absorbing boundary conditions prevailing at $a$ and $b$. 
(a)

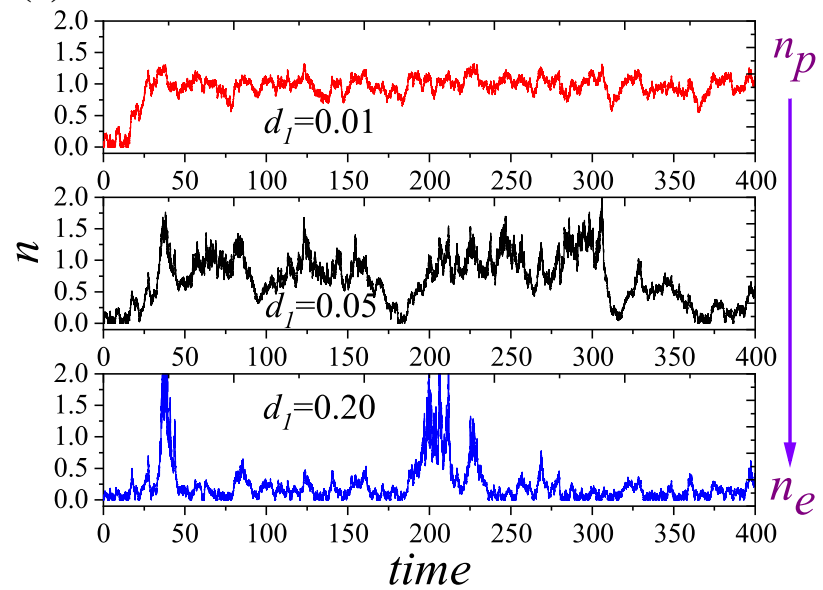

(b)

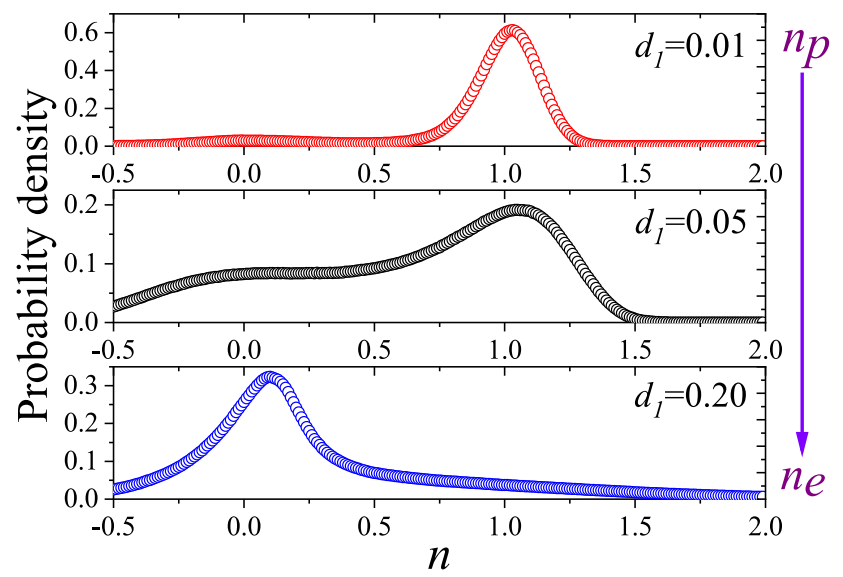

Fig. 2 Time series and probability density of steady-state $n(t)$ for different noise intensity $d_{1}: 0.01,0.05$, and 0.20 . The other parameter values are $\mu=0.2, \sigma=3.0, \lambda=1.425, d_{2}=0.01, q=0.8, \tau_{d}=0.1$. As the value of $d_{1}$ increases, the peak of $n_{e}$ state becomes higher, and the $n_{p}$ becomes lower

(a)

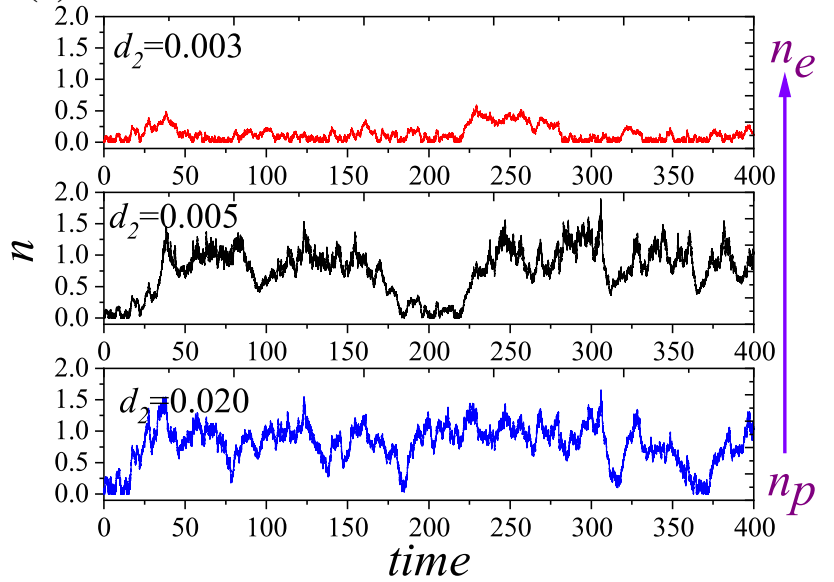

(b)

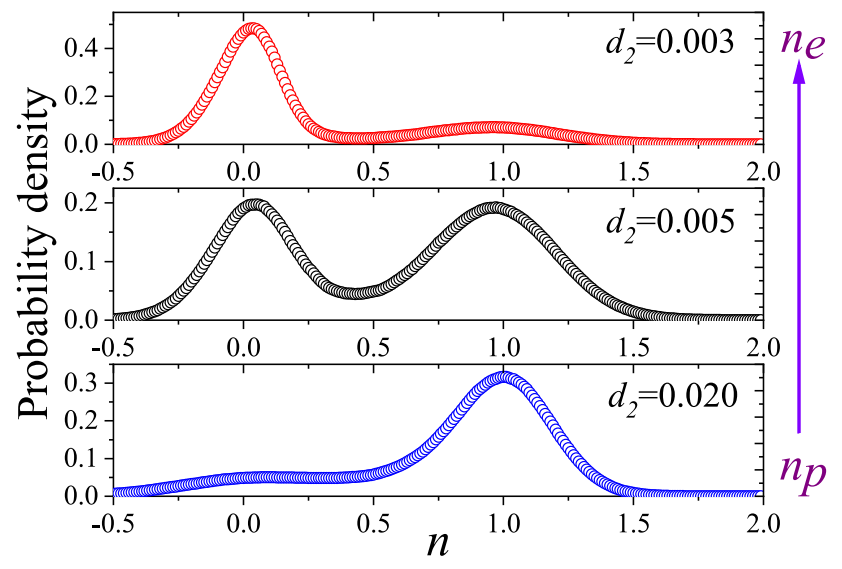

Fig. 3 Time series and probability density of steady-state $n(t)$ for different noise intensity $d_{2}: 0.003,0.005$, and 0.020 . The other parameter values are $\mu=0.2, \sigma=3.0, \lambda=1.425, d_{1}=0.03, q=0.8, \tau_{d}=0.1$. As the value of $d_{2}$ increases, the peak of $n_{p}$ state becomes higher, and the $n_{e}$ becomes lower

Thus, the STE can be given by

$$
T\left(n_{p} \rightarrow n_{e}\right)=\int_{n_{p}}^{n_{e}} \frac{\mathrm{d} n}{G\left(n, n_{\tau_{i}}\right) P_{s t}(n)} \int_{-\infty}^{n} P_{s t}(y) \mathrm{d} y
$$

Figure 9 shows the impact of the extrinsic noise intensity $d_{2}$ and cross-correlation intensity $q$ between two noises on the STE $\left(T\left(n_{p} \rightarrow n_{e}\right)\right)$, respectively. The STE exhibits one maximum value as $d_{1}$ increase is shown in Fig. 9a. As the extrinsic noise intensity $d_{2}$ increased, the maximum value is decreased. From Fig. 9b, we can see that when the cross-correlation intensity $q=0.1$, there is no peak in STE. If the cross-correlation intensity $q$ increased (0.8 and 0.9), the STE exhibits a nonmonotonic behavior with a maximum as $d_{1}$ increases. This maximum for STE as a function of $d_{1}$ identifies the characteristic of the noise-enhanced stability (NES) of the $n_{p}$ state. This nonmonotonic behavior was first found numerically by Hirsch et al. [70], and later by Dayan et al. [71], but without any physical explanation. Later, the phenomenon was observed experimentally by Mantegna and Spagnolo [9], who named it as NoiseEnhanced Stability (NES), having as signature a nonmonotonic behavior, with a maximum, of the average escape time from the metastable state as a function of the noise intensity. The NES phenomenon was theoretically explained and physically understood in the papers [72-74]. Recently, the investigation on the stabilizing effects of the noise was extended to the quantum context, studying the dissipative dynamics of a quantum particle moving along an asymmetric bistable potential [75]. This maximum for STE implies that the stability of the population survival state $n_{p}$ can be enhanced by the noises, and the mean lifetime of the population 
(a)

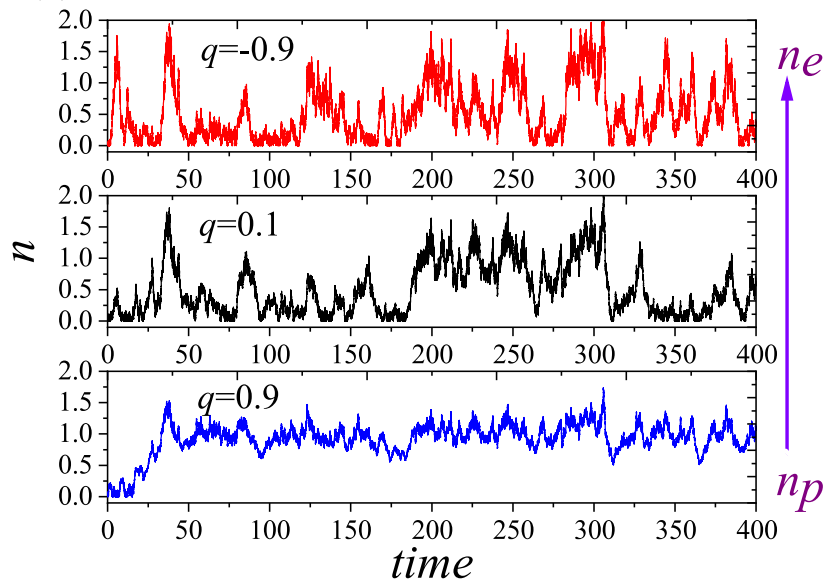

(b)

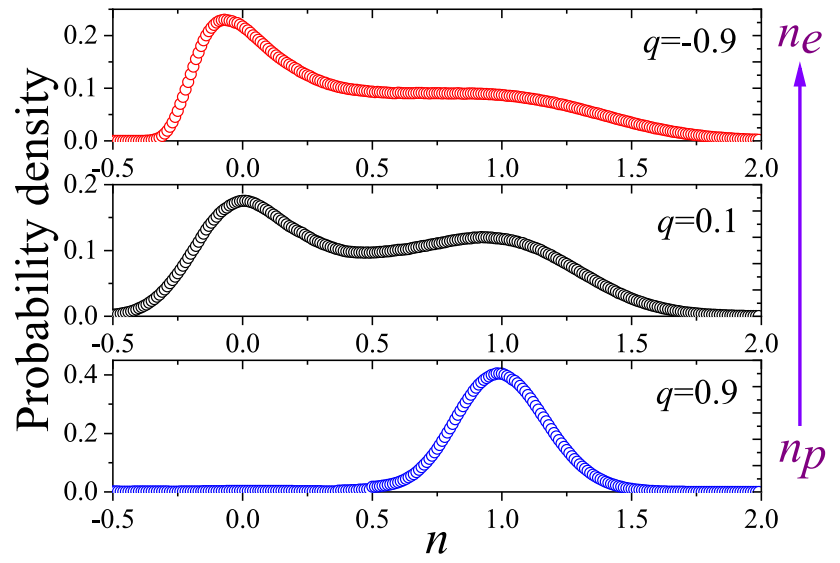

Fig. 4 Time series and probability density of steady-state $n(t)$ for different noise intensity $q=:-0.9,0.1$, and 0.9 . The other parameter values are $\mu=0.2, \sigma=3.0, \lambda=1.425, d_{1}=0.03, d_{2}=0.01, \tau_{d}=0.1$. As the value of $q=$ increases, the peak of $n_{p}$ state becomes higher, and the $n_{e}$ becomes lower

(a)

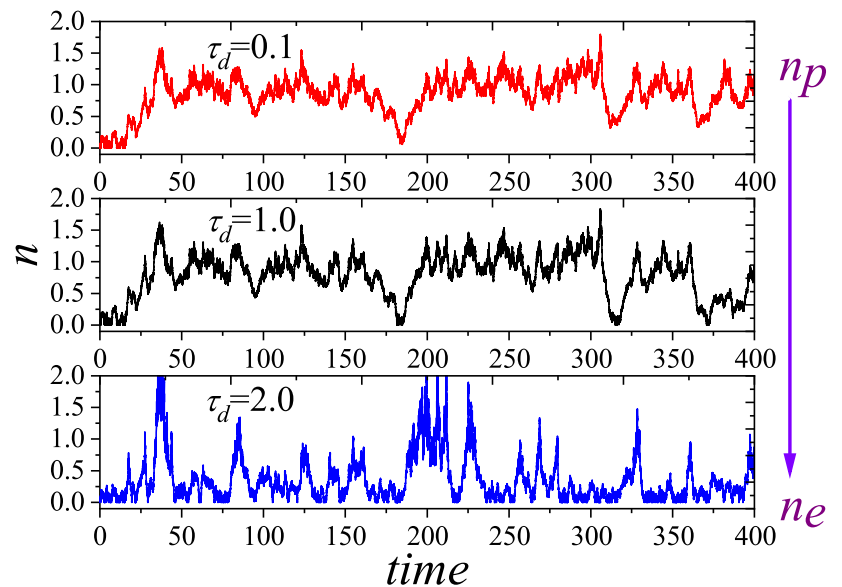

(b)

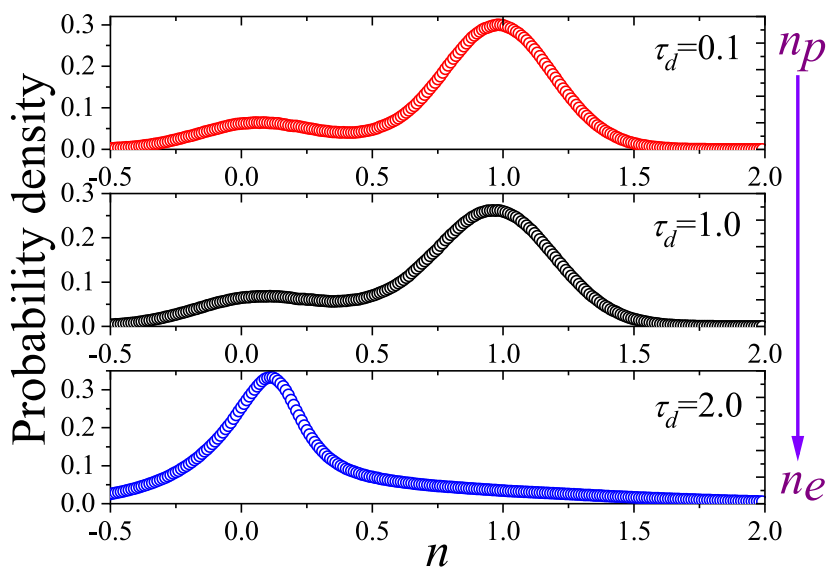

Fig. 5 The time series and the probability density of steady-state $n(t)$ for different time delays $\tau_{d}$ : 0.1, 1.0, and 2.0. The other parameter values are $\mu=0.2, \sigma=3.0, \lambda=1.425, d_{1}=0.03, d_{2}=0.01$, and $q=0.8$. As the value of $\tau_{d}$ increases, the peak of $n_{e}$ state becomes higher, and the $n_{p}$ becomes lower

survival state $n_{p}$ is longer than the deterministic decay time. Above results reveal that noise intensity leads to a $n_{p}$ state of expression and so it can be regarded as a control parameter of the shift time to extinction state $n_{e}$. This resonance-like behavior contradicts the monotonic behavior that was predicted by Kramers theory [76]. Simultaneously, the increase in $q$ lead to a increase in the STE (see Fig. 9b), i.e., the cross-correlation intensity can enhance stability of the population survival state $n_{p}$.

The impacts of the time delays $\tau_{d}, \tau_{b}$, and $\tau_{g}$ on the STE can be seen in Fig. 10, respectively. The STE first increases, reaches a maximum, and then decreases with increasing intrinsic noise intensity $d_{1}$, as shown in Fig. $10 \mathrm{a}-\mathrm{c}$. In other words, the STE as functions of the noise intensities $\left(d_{1}\right.$ exhibits a maximum, this maximum implies that the stability of the population survival state $n_{p}$ can be enhanced by the noise. The height of the maximum in the STE is decreased, is shown in Fig. 10a. On the other hand, the peak position is shifted to a small value of $d_{1}$ when the value of $\tau_{b}$ is increased in Fig. 10. While the height of its maximum is decreased and its position is shifted to a small value of $d_{1}$ when the value of $\tau_{g}$ is increased (see Fig. 10). It is emphasized that the increase of $\tau_{g}$ cannot still change STE, which is shown in Fig. 10c. The influence of $\tau_{g}$ on the STE of the system is the result of the interaction between $\tau_{d}$ and $\tau_{b}$. The maximum value not only decreases but also shifts to a position with less noise.

\subsection{Theoretical analysis and verification}

Here, we provide a theoretical analysis for the quasistationary probability distribution and shift time to extinction in the modified model I (i.e., Eq. (5)) with 
(a)

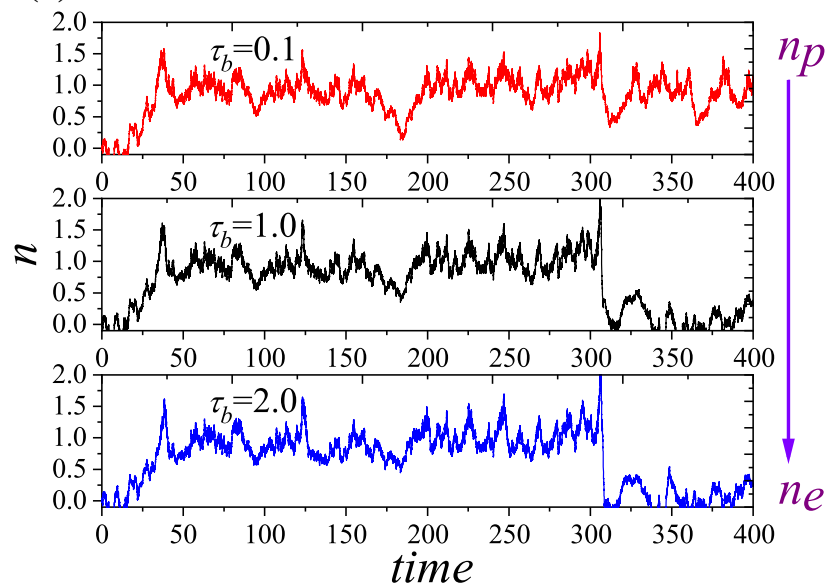

(b)

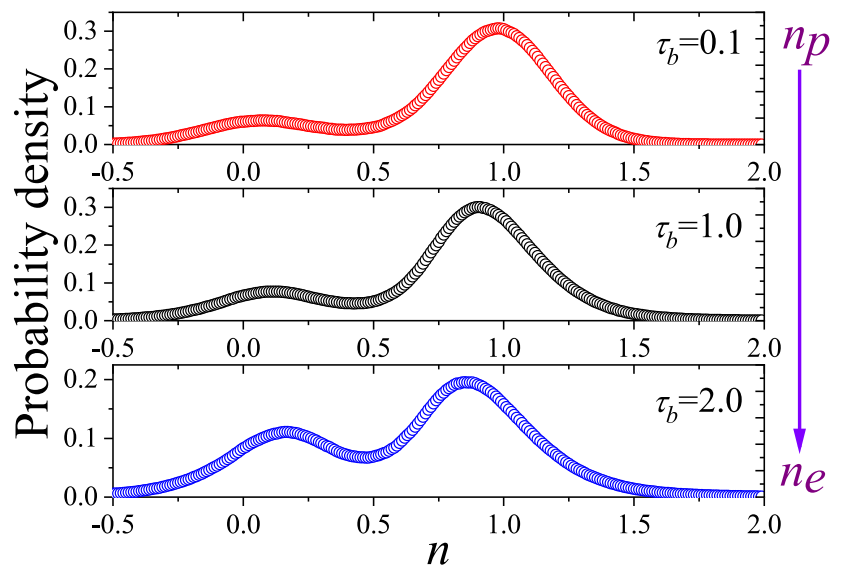

Fig. 6 The time series and the probability density of steady-state $n(t)$ for different time delays $\tau_{b}: 0.1,1.0$, and 2.0 . The other parameter values are $\mu=0.2, \sigma=3.0, \lambda=1.425, d_{1}=0.03, d_{2}=0.01$, and $q=0.8$. As the value of $\tau_{b}$ increases, the peak of $n_{e}$ state becomes higher, and the $n_{p}$ becomes lower

(a)

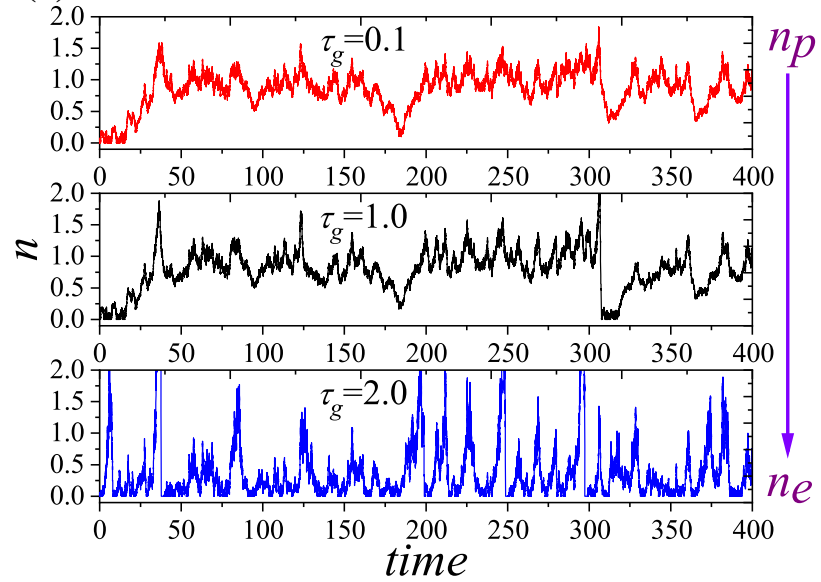

(b)

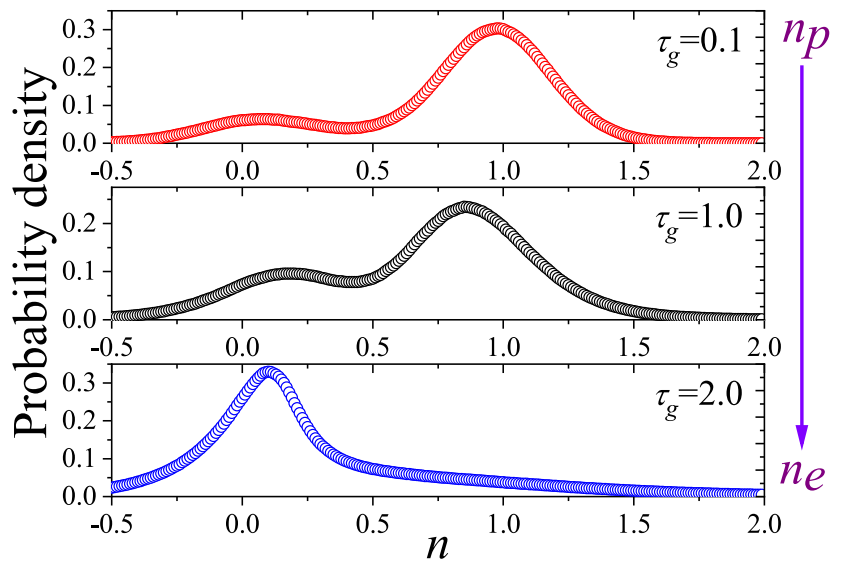

Fig. 7 The time series and the probability density of steady-state $n(t)$ for different time delays $\tau_{g}: 0.1,1.0$, and 2.0 . The other parameter values are $\mu=0.2, \sigma=3.0, \lambda=1.425, d_{1}=0.03, d_{2}=0.01$, and $q=0.8$. As the value of $\tau_{g}$ increases, the peak of $n_{e}$ state becomes higher, and the $n_{p}$ becomes lower

environmental noises and time delay $\tau_{d}$. For this case, the effective drift and diffusion coefficients $F_{\text {eff }}(n)$ and $G_{\text {eff }}(n)$ are given by

$$
\begin{aligned}
F_{\text {eff }}(n)= & \sqrt{\frac{1}{2 \pi G^{(0)}(n) \tau_{d}}} \int_{-\infty}^{\infty} F\left(n, n_{\tau_{d}}\right) \\
& \exp \left\{-\frac{\left[n_{\tau_{d}}-n-h^{(0)}(n) \tau_{d}\right]^{2}}{2 G^{(0)}(n) \tau_{d}}\right\}, \\
G_{\text {eff }}(n)= & \sqrt{\frac{1}{2 \pi G^{(0)}(n) \tau_{d}}} \int_{-\infty}^{\infty} G\left(n, n_{\tau_{d}}\right) \\
& \exp \left\{-\frac{\left[n_{\tau_{d}}-n-h^{(0)}(n) \tau_{d}\right]^{2}}{2 G^{(0)}(n) \tau_{d}}\right\}
\end{aligned}
$$

with

$$
\begin{aligned}
& F\left(n, n_{\tau_{d}}\right)=h\left(n, n_{\tau_{d}}\right)+\frac{1}{2} G^{\prime}\left(n, n_{\tau_{d}}\right), \\
& G\left(n, n_{\tau_{d}}\right)=d_{1} g_{1}^{2}\left(n, n_{\tau_{d}}\right)+2 q \sqrt{d_{1} d_{2}} g_{1}\left(n, n_{\tau_{d}}\right)+d_{2} .
\end{aligned}
$$

and $G^{(0)}(n)=\left.G\left(n, n_{\tau_{d}}\right)\right|_{\left(n_{\tau_{d}}=n\right)}=d_{1} n^{2}-2 q \sqrt{d_{1} d_{2}} n+$ $d_{2}, h^{(0)}(n)=\left.h\left(n, n_{\tau_{d}}\right)\right|_{\left(n_{\tau_{d}}=n\right)}=-\frac{\sigma}{6} n^{3}+\frac{\lambda}{2} n^{2}-\mu n$. Substituting equations $(15,16)$ into $(13,14)$. We obtain

$$
\begin{aligned}
F_{\text {eff }}(n)= & \left(1-\mu \tau_{d}\right)\left(-\frac{\sigma}{6} n^{3}+\frac{\lambda}{2} n^{2}-\mu n\right) \\
& +d_{1}\left(1-\tau_{d}\right)^{2} n-q \sqrt{d_{1} d_{2}}\left(1-\tau_{d}\right), \\
G_{\text {eff }}(n)= & d_{1}\left(1-\tau_{d}\right)^{2} n^{2}-2 q \sqrt{d_{1} d_{2}}\left(1-\tau_{d}\right) n+d_{2} .
\end{aligned}
$$



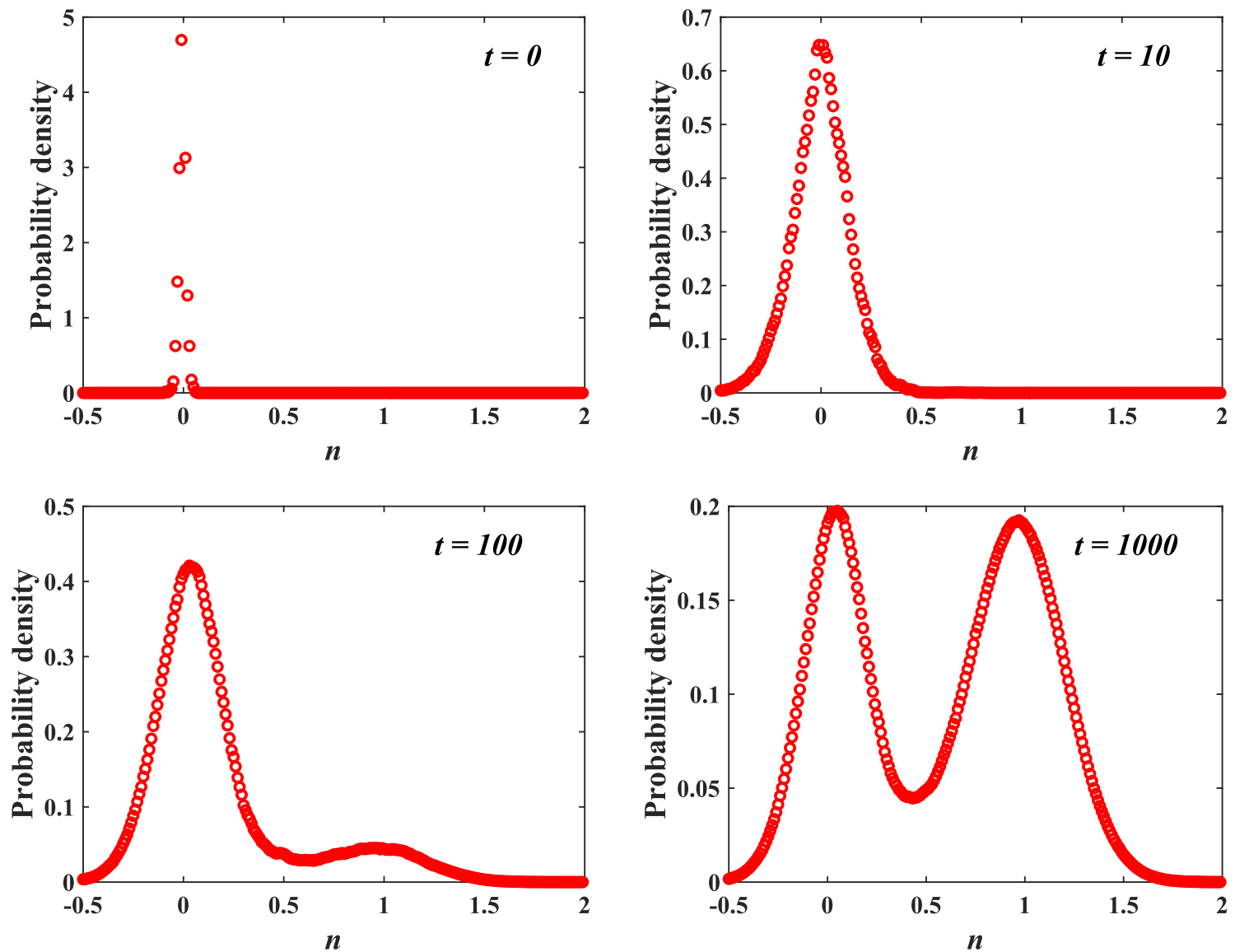

Fig. 8 Probability density of $n(t)$ for the different times $t=0, t=10, t=100$, and $t=1000$. The other parameter values are $\mu=0.2, \sigma=3.0, \lambda=1.425, d_{1}=0.03, d_{2}=0.005, q=0.8$, and $\tau_{d}=0.1$

From Eq. (8), the quasi-stationary probability distribution function (PDF) of populations can be derived as

$$
\begin{aligned}
P_{s t}(n) & =\frac{N}{\sqrt{G_{\mathrm{eff}}(n)}} \exp \int^{n} \frac{h_{\mathrm{eff}}\left(n^{\prime}\right)}{G_{\mathrm{eff}}\left(n^{\prime}\right)} \mathrm{d} n^{\prime} \\
& =\frac{N}{\sqrt{G_{\mathrm{eff}}(n)}} \exp \left[-\frac{U_{\mathrm{eff}}(n)}{d_{1}}\right]
\end{aligned}
$$

where $N$ is a normalization constant and $U_{\text {eff }}(n)$ is the effective potential function, which can be expressed exactly as:

$U_{\text {eff }}(n)=-d_{1} \int^{n} \frac{\left(1-\mu \tau_{d}\right)\left(-\frac{\sigma}{6} n^{\prime 3}+\frac{\lambda}{2} n^{\prime 2}-\mu n^{\prime}\right)}{d_{1}\left(1-\tau_{d}\right)^{2} n^{\prime 2}-2 q \sqrt{d_{1} d_{2}}\left(1-\tau_{d}\right) n^{\prime}+d_{2}} \mathrm{~d} n^{\prime}$

Integrating Eq. (20), we obtain

$$
\begin{aligned}
U_{\mathrm{eff}}(n)= & \beta_{1} n^{2}+\beta_{2} n \\
& +\beta_{3} \ln \left|\left(1-\tau_{d}\right)^{2} n^{2}-2\left(1-\tau_{d}\right) q \sqrt{d} n+d\right| \\
& +\frac{\beta_{4}}{\left(1-\tau_{d}\right) \sqrt{d\left(1-q^{2}\right)}} \arctan \frac{\left(1-\tau_{d}\right) n-q \sqrt{d}}{\sqrt{d\left(1-q^{2}\right)}}
\end{aligned}
$$

with

$$
\begin{aligned}
d= & d_{2} / d_{1}, \\
\beta_{1}= & \frac{\sigma\left(1-\mu \tau_{d}\right)}{12\left(1-\tau_{d}\right)^{2}}, \\
\beta_{2}= & \frac{\sigma q \sqrt{d}\left(1-\mu \tau_{d}\right)}{3\left(1-\tau_{d}\right)^{3}}-\frac{\lambda\left(1-\mu \tau_{d}\right)}{2\left(1-\tau_{d}\right)^{2}}, \\
\beta_{3}= & {\left[\frac{\sigma q \sqrt{d}\left(1-\mu \tau_{d}\right)}{3\left(1-\tau_{d}\right)}-\frac{\lambda}{2}\right] \frac{q \sqrt{d}\left(1-\mu \tau_{d}\right)}{\left(1-\tau_{d}\right)^{3}} } \\
& +\left[\mu-\frac{\sigma d}{6\left(1-\tau_{d}\right)^{2}}\right] \frac{1-\mu \tau_{d}}{2\left(1-\tau_{d}\right)^{2}}, \\
\beta_{4}= & {\left[\mu-\frac{\sigma d}{6\left(1-\tau_{d}\right)^{2}}\right] \frac{q \sqrt{d}\left(1-\mu \tau_{d}\right)}{\left(1-\tau_{d}\right)} } \\
& +\left[\frac{\sigma q \sqrt{d}}{3\left(1-\tau_{d}\right)}-\frac{\lambda}{2}\right] \frac{d\left(2 q^{2}-1\right)\left(1-\mu \tau_{d}\right)}{\left(1-\tau_{d}\right)^{2}} .
\end{aligned}
$$

In addition, the impacts of noises and time delay on the bifurcation diagram are given by the maxima of the PDF. The maxima of the PDF are obtain from the general equation $F_{\text {eff }}(n)-G_{\text {eff }}^{\prime}(n)=0$. According to equations $(13,14)$, this leads to 
(a)

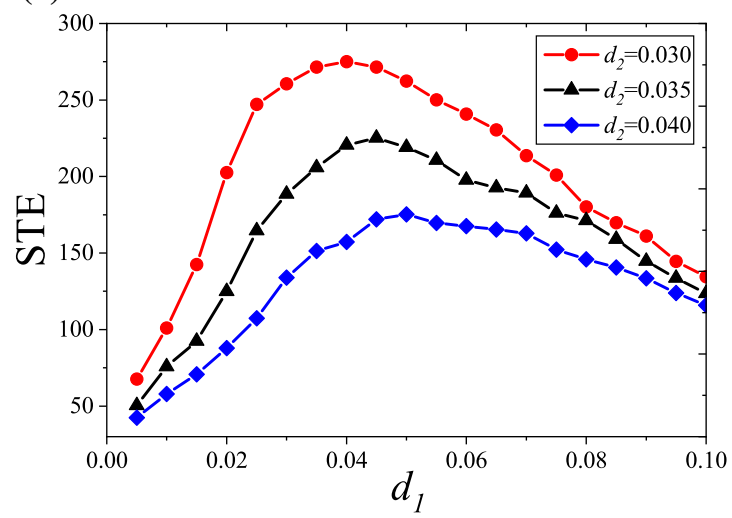

(b)

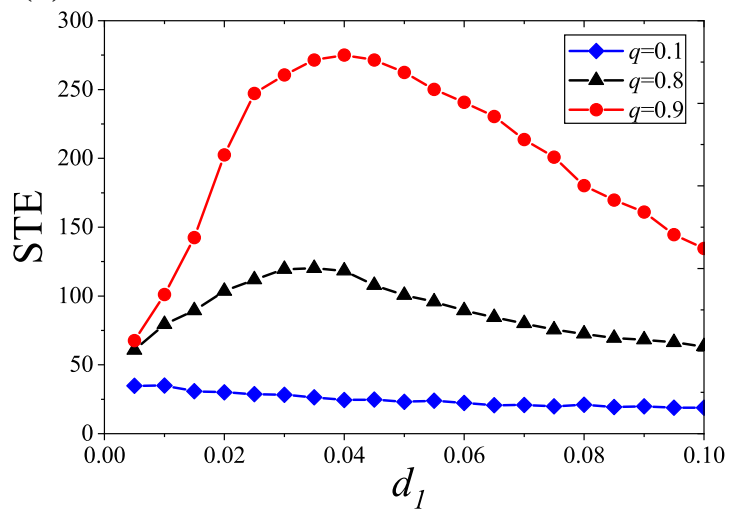

Fig. 9 The STE as a function of the intrinsic noise intensity $d_{1}$ and for different values of extrinsic noise intensity $d_{2}$ and cross-correlation intensity $q . q=0.9$ in (a) and $d_{2}=0.1$ in (b). The other parameter value are $\mu=0.2, \sigma=3.0, \lambda=1.425$, and $\tau_{d}=0.1$

$$
\begin{aligned}
& \left(1-\mu \tau_{d}\right)\left(-\frac{\sigma}{6} n^{3}+\frac{\lambda}{2} n^{2}-\mu n\right)-d_{1}\left(1-\tau_{d}\right)^{2} n \\
& \quad+q \sqrt{d_{1} d_{2}}\left(1-\tau_{d}\right)=0
\end{aligned}
$$

Using the steepest descent method, the explicit expression for the SET (12) is given by

$$
\begin{aligned}
& T\left(n_{p} \rightarrow n_{e}\right) \\
& \quad=\frac{2 \pi}{\sqrt{\left|U^{\prime \prime}\left(n_{r}\right) U^{\prime \prime}\left(n_{p}\right)\right|}} \exp \left[\frac{U_{\mathrm{eff}}\left(n_{r}\right)-U_{\mathrm{eff}}\left(n_{p}\right)}{d_{1}}\right]
\end{aligned}
$$

where $U(n)$ and $U_{\text {eff }}(n)$ are given by Eqs. (2) and (21), respectively.

To check the credibility of the numerical simulations in population system subject to the noises and time delay, let us compare the numerical simulations (Figs. 2, 3, 4, 5) with approximate theoretical results (Fig. 11a-d). Here, we provide a theoretical analysis for the stationary probability distribution in population system with time delay $\tau_{d}$. The numerical simulations in the probability distributions are consistent with the approximate theoretical results, which implies that the numerical simulations in population system with time delays and noises are credible.

The phenomenon of noise-induced transition [77] and phase transition $[78,79]$ have been shown in other nonlinear systems, here the noise-induced transition exists in the population system. The impacts of the extrinsic noise intensity $d_{2}$ on the bifurcation diagram can be seen in Fig. 12a through equation (21). When the extrinsic noise intensity is $\operatorname{small}\left(d_{2}=0.003\right)$ and $\mu_{1}<$ $\mu<\mu_{2}$, the Eq. (21) has three roots. In other words, the system corresponding SPD is a bimodal structure. As the value of $d_{2}$ increases $\left(d_{2}=0.030\right)$ and $\mu_{1}<\mu<\mu_{1}^{\prime \prime}$, the Eq. (21) has one root and the corresponding SPD is a unimodal structure. However, if the value of $\mu$ increase, we can see that the structure of the
SPD is changed from unimodal to bimodal when $d_{2}$ is increased.

Similarly, it is shown from Fig. $12 \mathrm{~b}$ that the impacts of the time delay $\tau_{d}$ on the SPD and bifurcation diagram through equations (21). When the $\mu \in \Delta \mu_{1}$ and $\tau_{d}=0.1$, equation (21) has one root and the corresponding SPD has an un unimodal structure, as shown in Fig. 12b. As the value of $\tau_{d}$ increasing ( see $\tau_{d}=0.9$ in Fig. 12b), Eq. (21) has three roots and the corresponding SPD is a bimodal structure, i.e., the structure of the SPD is changed from unimodal to bimodal when $\tau_{d}$ is increased. Therefore, the impacts of the time delay on the SPD and bifurcation diagram are consistent.

The three-dimensional curves of the STE as functions of $n$ and noises (or time delay) are shown in Fig. 13, respectively. Obviously, the STE as functions of the noise intensities $d_{1}$ exhibits a maximum, this maximum implies that the stability of the population state can be enhanced by the noise [9-13,64,65]. When the value of $d_{2}$ is larger, the peak value is higher (Fig. 13a). In Fig. 13b, we can see that the cross-correlation intensity $q$ has a great influence on the change of STE. When the value of $q$ increases, the maximum value of STE increases significantly. The time delay $\tau_{d}$ promotes the increase of the maximum value of STE, as shown in Fig. 13c. These results can also correspond well with numerical simulation results, as shown in Figs. 9 and 10.

\subsection{The SR}

We consider a simple periodic form $\widetilde{A} \cos \omega t, A$ and $\omega$ are amplitude and frequency of periodic signal, respectively. Therefore, Eq. (5) can be rewritten as

$$
\frac{\mathrm{d} n}{\mathrm{~d} t}=-\frac{\sigma}{6} n^{3}+\frac{\lambda}{2} n^{2}-\left(\mu+\xi_{1}(t)\right) n_{\tau_{d}}+\xi_{2}(t)+\widetilde{A} \cos \omega t
$$

To investigate the $\mathrm{SR}$ in a population system, we need the SNR of the system. First, we derive transition 
(a)

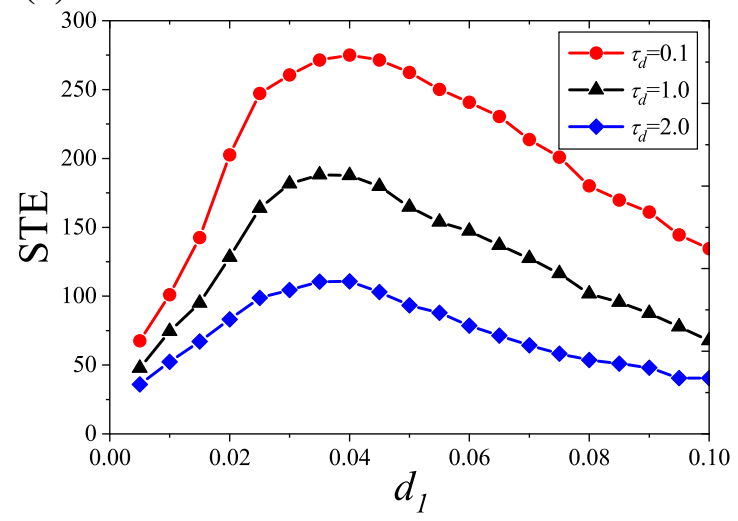

(b)

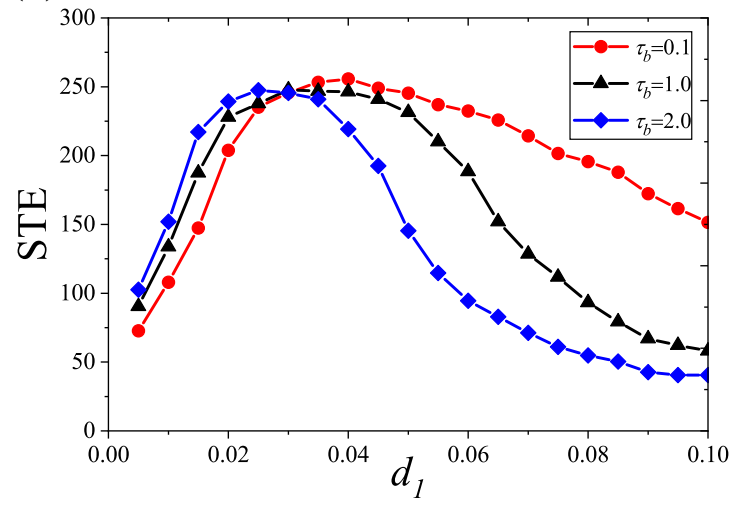

(c)

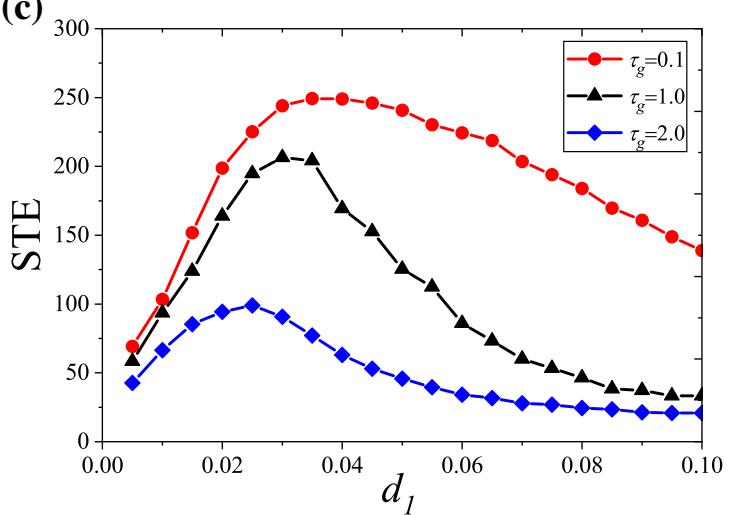

Fig. 10 The STE as a function of $d_{1}$ with different time delays $\tau_{d}(\mathbf{a}), \tau_{b}(\mathbf{b})$, and $\tau_{g}(\mathbf{c})$. The other parameter value are $\mu=0.2, \sigma=3, \lambda=1.425, q=0.9$, and $d_{2}=0.1$

rates between two states and then calculate the SNR of the system. Using the steepest descent method [68], the explicit expression for the (STE) $T_{1,2}$ of the process $n(t)$ to reach the state $n_{p, e}$ with initial condition $n_{e, p}$ is given by

$$
\begin{aligned}
T_{n_{p}-n_{e}}= & T_{1}=\frac{2 \pi}{\sqrt{\left|U^{\prime \prime}\left(n_{r}\right) U^{\prime \prime}\left(n_{p}\right)\right|}} \\
& \exp \left[\frac{U_{\text {eff }}\left(n_{r}, t\right)-U_{\text {eff }}\left(n_{p}, t\right)}{d_{1}}\right], \\
T_{n_{e}-n_{p}}= & T_{2}=\frac{2 \pi}{\sqrt{\left|U^{\prime \prime}\left(n_{r}\right) U^{\prime \prime}\left(n_{e}\right)\right|}} \\
& \exp \left[\frac{U_{\text {eff }}\left(n_{r}, t\right)-U_{\text {eff }}\left(n_{e}, t\right)}{d_{1}}\right] .
\end{aligned}
$$

Note that the above result is valid only when the intensity of two types of noise, measured by $d_{1}$ and $d_{2}$, is small in comparison with the energy barrier height: $d_{1}, d_{2}<\Delta U=\left|U\left(n_{r}, t\right)-U\left(n_{p, e}, t\right)\right|$. This provides restriction on the parameters (i.e., $d_{1}, d_{2}, \widetilde{A}, \omega$, et $a l$.). We must point out that the following results are restricted in valid regions. Therefore, we can obtain the transition rates

$$
\begin{aligned}
W_{n_{p}-n_{e}}= & W_{1}=\frac{\sqrt{\left|U^{\prime \prime}\left(n_{r}\right) U^{\prime \prime}\left(n_{p}\right)\right|}}{2 \pi} \\
& \exp \left[\frac{U_{\mathrm{eff}}\left(n_{p}, t\right)-U_{\mathrm{eff}}\left(n_{r}, t\right)}{d_{1}}\right], \\
W_{n_{e}-n_{p}}= & W_{2}=\frac{\sqrt{\left|U^{\prime \prime}\left(n_{r}\right) U^{\prime \prime}\left(n_{e}\right)\right|}}{2 \pi} \\
& \exp \left[\frac{U_{\mathrm{eff}}\left(n_{e}, t\right)-U_{\mathrm{eff}}\left(n_{r}, t\right)}{d_{1}}\right] .
\end{aligned}
$$

where $U^{\prime \prime}$ is the second derivative of $U$ with respect to $n$ and the $U(n)$ is given by Eq. (2). The $U_{\text {eff }}(n, t)$ is to rewrite $U_{\text {eff }}(n, t)$ [Eq.(20)] after considering the periodic signal, the method is as in Ref. [45].

We start by considering a system described by a discrete random dynamical variable $n$ that adopts two possible values: $n_{p}$ and $n_{e}$, with probabilities $n_{p, e}$, respectively. The probabilities satisfy the condition $n_{1}+n_{2}=$ 1. The master equation for our problem is

$$
\begin{aligned}
\frac{\mathrm{d} n_{1}}{\mathrm{~d} t} & =-\frac{\mathrm{d} n_{2}}{\mathrm{~d} t}=W_{2}(t) n_{2}(t)-W_{1}(t) n_{1}(t) \\
& =W_{2}(t)-\left[W_{2}(t)+W_{1}(t)\right] n_{1}(t)
\end{aligned}
$$



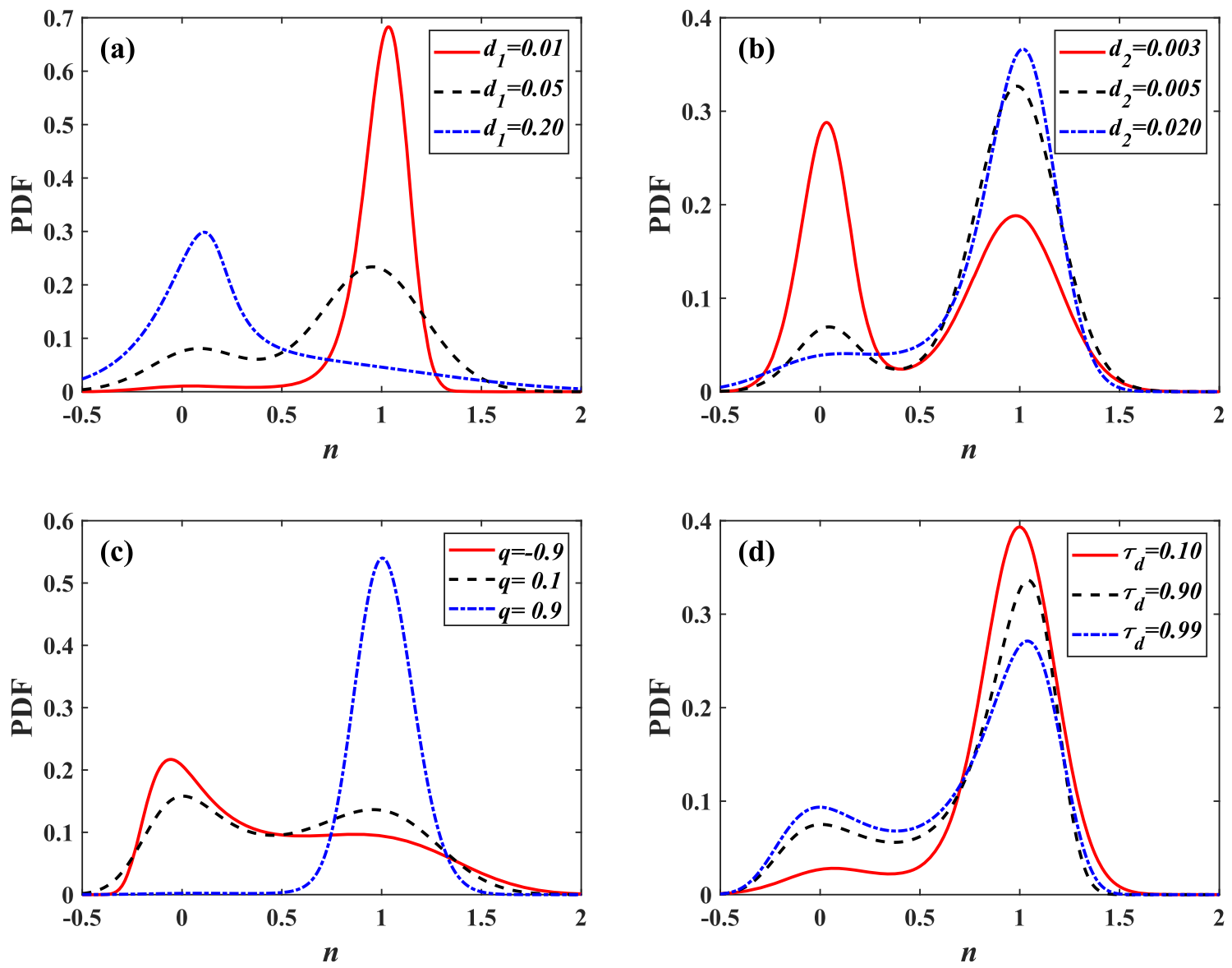

Fig. 11 The steady-state probability distribution of population as a function of $n(t)$ for different $d_{1}$ (a), extrinsic noise intensities $d_{2}(\mathbf{b})$, cross-correlation intensities $q$ between two noises (c), and time delay $\tau_{d}(\mathbf{d})$. The other parameter values are $\mu=0.2, \sigma=3, \lambda=1.425, \mathbf{a} d_{2}=0.01, q=0.8, \tau_{d}=0.1 ; \mathbf{b} d_{1}=0.03, q=0.8$ and $\tau_{d}=0.1 ; \mathbf{c} d_{1}=0.03, d_{2}=0.01$ and $\tau_{d}=0.1 ; \mathbf{d} d_{1}=0.03, d_{2}=0.01$ and $q=0.8$

(a)

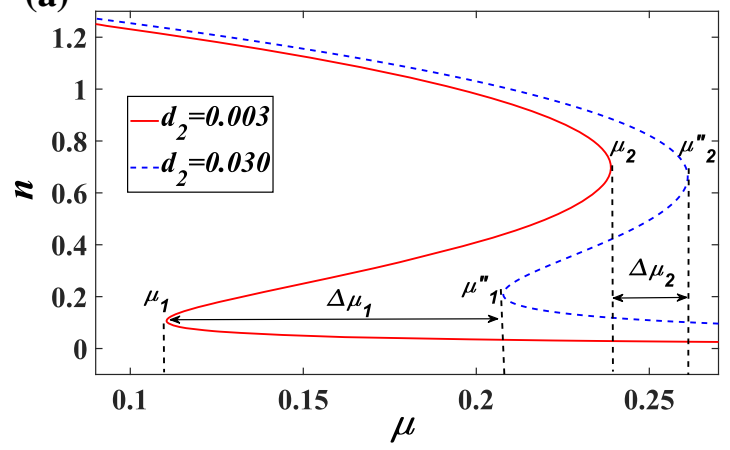

(b)

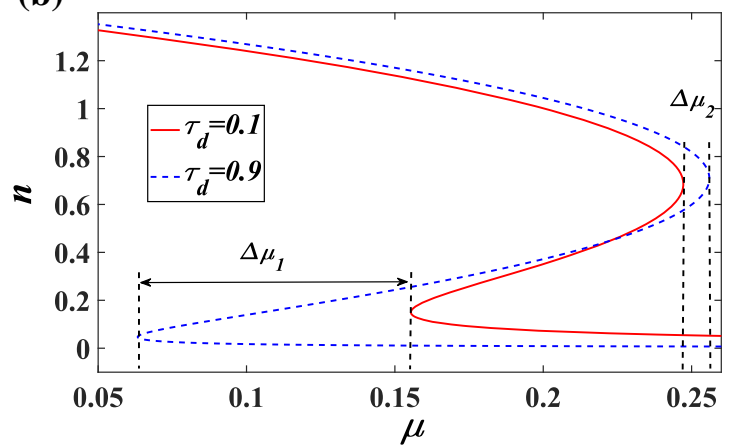

Fig. 12 Bifurcation diagram for the protein concentration $n$ as a function of $\mu$ for different extrinsic noise intensities $d_{2}$ (a) and different time delay $\tau_{d}(\mathbf{b})$. The other parameter values are $\sigma=3, \lambda=1.425$, a $d_{1}=0.03, q=0.8, \tau_{d}=0.1 ; \mathbf{b}$ $d_{1}=0.03, d_{2}=0.03, q=0.8$ 

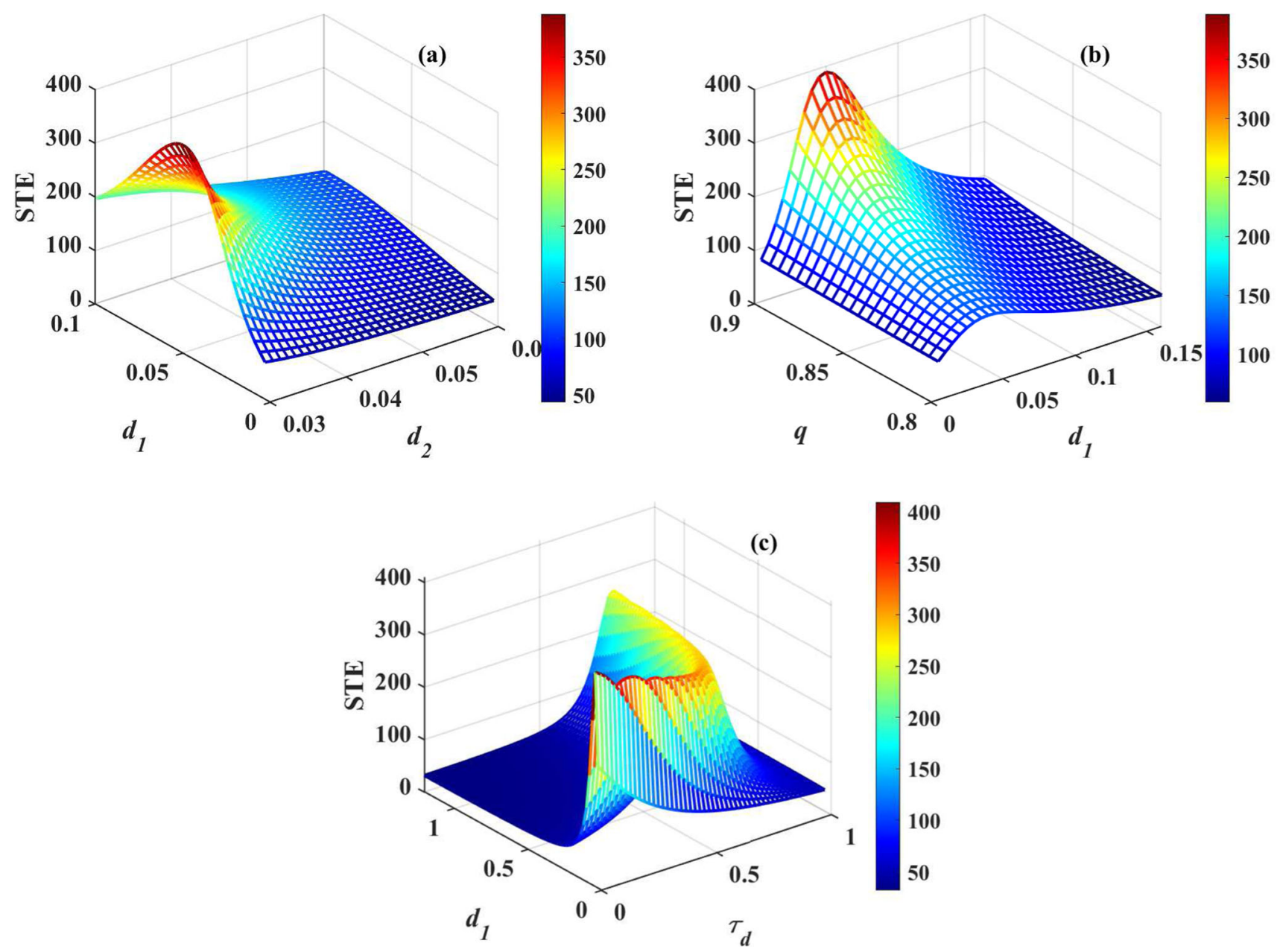

Fig. 13 Three-dimensional curve of the STE affected by different noises and time delay. The parameter values are $\mu=0.2$, $\sigma=3.0, \lambda=1.425, \mathbf{a} q=0.8, \tau_{d}=0.1 ; \mathbf{b} d_{2}=0.03 ; \tau_{d}=0.1 ; \mathbf{c} d_{2}=0.03, q=0.8$

where $W_{1,2}$ are the transition rates out of the $n_{p, e}$ states. Since we assume the signal amplitude is small enough (i.e., $\widetilde{A} \ll 1$ ), the transition rates $W_{1,2}(t)$ can be expanded up to the first order of $\widetilde{A}$ as

$$
\begin{aligned}
& W_{1}(t)=\mu_{1}-\nu_{1} \widetilde{A} \cos \omega t, \\
& W_{2}(t)=\mu_{2}+\nu_{2} \widetilde{A} \cos \omega t,
\end{aligned}
$$

where

$$
\begin{aligned}
& \mu_{1}=\left.W_{1}\right|_{S(t)=0}, \quad \nu_{1}=-\left.\frac{\mathrm{d} W_{1}}{\mathrm{~d} S(t)}\right|_{S(t)=0}, \quad S(t)=\widetilde{A} \cos \omega t \\
& \mu_{2}=\left.W_{2}\right|_{S(t)=0}, \quad \nu_{2}=\left.\frac{\mathrm{d} W_{2}}{\mathrm{~d} S(t)}\right|_{S(t)=0}
\end{aligned}
$$

Then, the SNR in terms of the output signal power spectrum can be given by

$$
\mathrm{SNR}=\frac{\widetilde{A}^{2} \pi\left(\nu_{2} \mu_{1}+\nu_{1} \mu_{2}\right)^{2}}{4 \pi \mu_{1} \mu_{2}\left(\mu_{1}+\mu_{2}\right)}
$$

By virtue of the expression of SNR [Eq. (33)] as a function of intrinsic noise intensity $d_{1}$ and the crosscorrelation intensity $q$, time delay $\tau_{d}$ are plotted in the following Fig. 14a, b. In Fig. 14a, SNR as a function of $d_{1}$ exhibits a maximum for the negative value of $q$. The existence of the maximum in the SNR as a function of $d_{1}$ are the identifying characteristics of the SR phenomenon. As the value of $q$ is continues increasing, the maximum in the SNR as a function of $d_{1}$ is decreased, i.e., the positive cross-correlation intensity between two noises weakens the SR phenomenon. In Fig. $14 \mathrm{~b}$, as value of $\tau_{d}$ increases, maximum in SNR as a function of $d_{1}$ decreases. Similarly, the time delay $\tau_{d}$ also weakens the SR phenomenon.

The SNR as a function of extrinsic noise intensity $d_{2}$ and the cross-correlation intensity $q$, time delay $\tau_{d}$ are plotted in Fig. 15a, b. Figure 15 shows that SNR as a function of $d_{2}$ exhibits only a maximum. The maximum in SNR as a function of $d_{2}$ is decreased as value of $q$ and $\tau_{d}$ increase, i.e., cross-correlation intensity $q$ and time delay $\tau_{d}$ weaken SR phenomenon. In Fig. 16, the SNR as a function of intrinsic noise intensity $d_{1}$ and extrinsic noise intensity $d_{2}$ are plotted for different value of the 

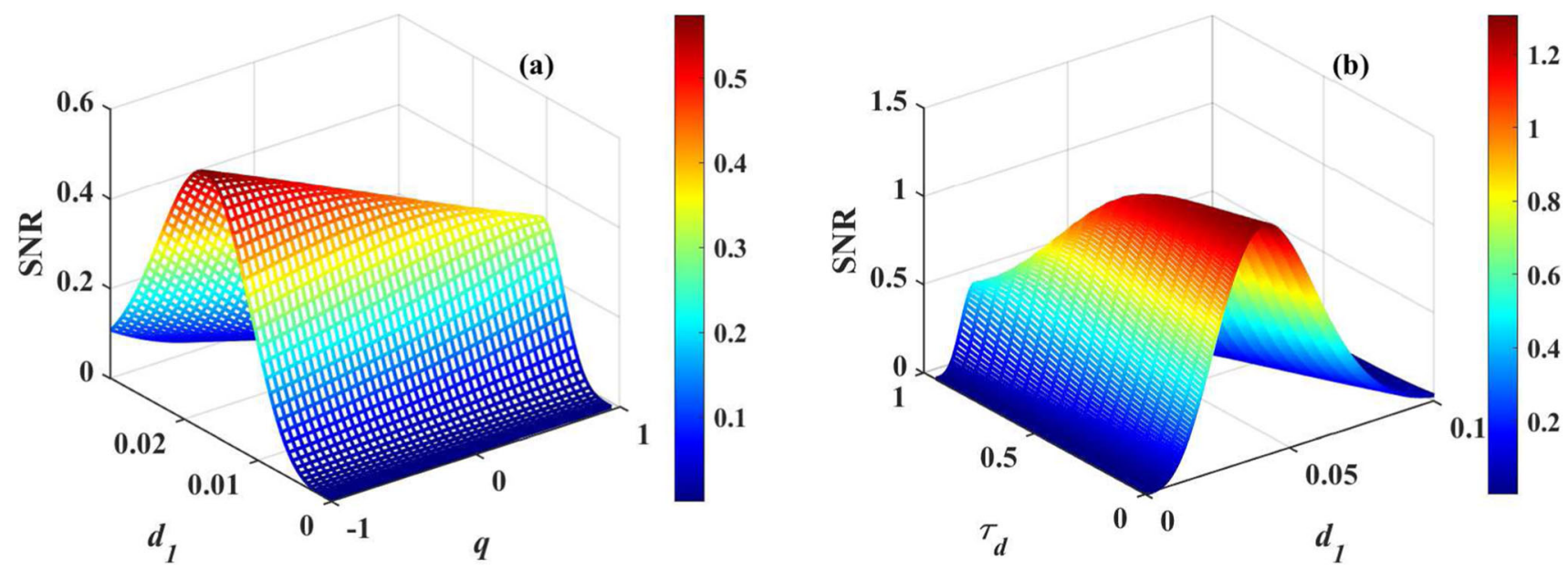

Fig. 14 The SNR as a function of intrinsic noise intensity $d_{1}$ for cross-correlation intensity $q(\mathbf{a})$ and time delay $\tau_{d}(\mathbf{b})$. The other parameter values are $\mu=0.2, \sigma=3.0, \lambda=1.425, d_{2}=0.001, A=0.1, \mathbf{a} \tau_{d}=0.9 ; \mathbf{b} q=-0.5$
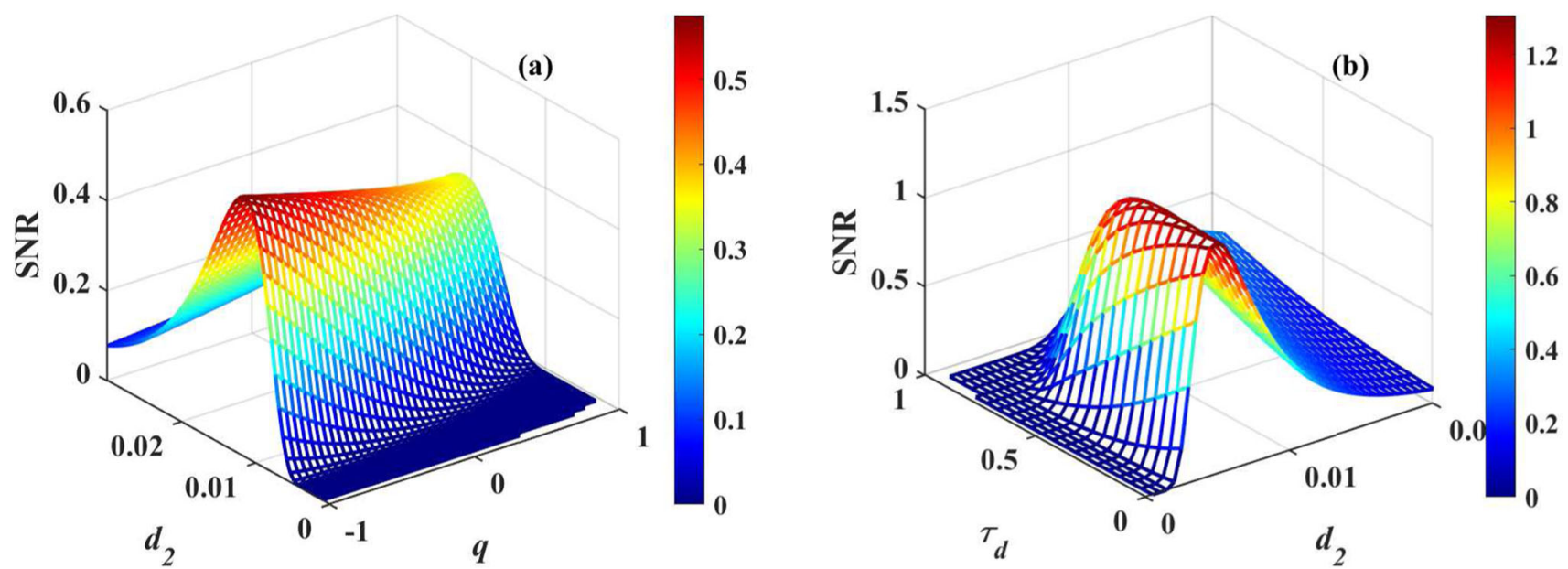

Fig. 15 The SNR as a function of extrinsic noise intensity $d_{2}$ for cross-correlation intensity $q$ (a) and time delay $\tau_{d}$ (b). The other parameter values are $\mu=0.2, \sigma=3.0, \lambda=1.425, d_{1}=0.2, A=0.1, \mathbf{a} \tau_{d}=0.9 ; \mathbf{b} q=-0.5$
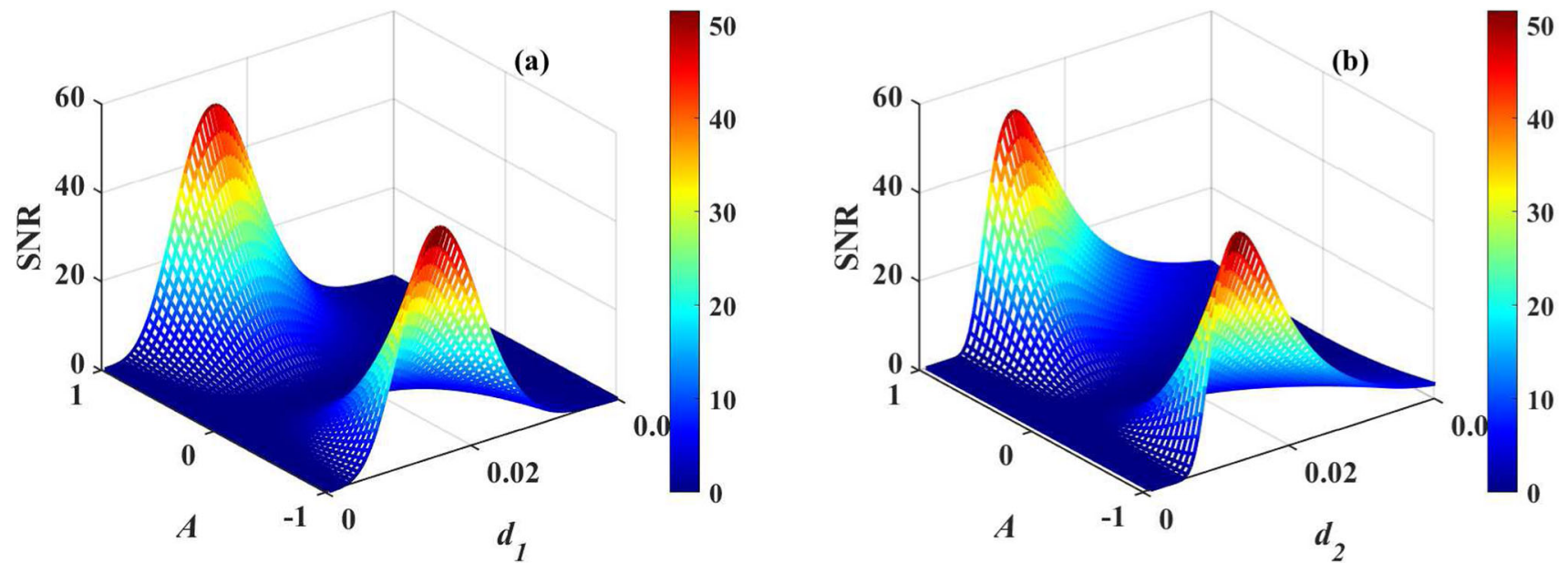

Fig. 16 The SNR as a function of signal amplitude $A$ for intrinsic noise intensity $d_{1}(\mathbf{a})$ and extrinsic noise intensity $d_{2}$ (b). The other parameter values are $\mu=0.2, \sigma=3.0, \lambda=1.425, \tau_{d}=0.9, q=-0.5$, a $d_{2}=0.001 ; \mathbf{b} d_{1}=0.2$ 
signal amplitude $A$. The interesting point here is that, when the absolute value of signal amplitude $A$ increases, a maximum value appears. If the value of $A$ is close to 0 , the maximum value disappears. In other words, the signal amplitude $|A|$ enhances the SR phenomenon.

\section{Conclusions}

The main point of our work is that if we consider the three different time delays, e.g., model I, model II and model III, then noises and time delays induce regime shifts between the two alternative stable states, typically the shift process can be further accelerated with increasing time delay. The deterministic potential related to the deterministic force of Eq. (2) has two steady stable states, which correspond to the population state and the extinction state, respectively. We are interested in how the shift from one state to the other on account of the noises and time delayed. The main finding is that the $d_{1}$ or $\tau_{d}$ can induce the shift from $n_{p}$ state to $n_{e}$ one. In addition, the time delay $\tau_{b}$ and $\tau_{g}$ also promote the transition from the $n_{p}$ state to the $n_{e}$ state. However, the opposite is that as the $d_{2}$ or $q$ increases, the $n_{e}$ state will switch to $n_{p}$ state. In other words, when the $d_{2}$ or $q$ increases, enhances the probability density of $n_{p}$ state. To explore the mechanism of transformation between the two states, we have also studied STE of populations. The main finding is that the STE as a function of the noise intensity $\left(d_{1}\right)$ can exhibit a maximum, which indicates the existence of an appropriate noise intensity leading to a maximal STE. This nonmonotonic behavior is a signature of the noise enhanced stability phenomenon (NES) observed in many physical and complex metastable systems [64] and here in a population dynamics model, in the presence of time delay and environmental noise sources. In particular, the maximum for STE increases (or decreases) as $q$ (or $\tau_{d}$ ) increases. It was demonstrated that a shift process can be induced by the noises $d_{1}$ and $d_{2}$, cross-correlation intensity $q$, time delay $\tau_{d}$. Furthermore, effects of the cross-correlations intensity, time delay and signal intensity on SNR as a function of noise intensities are analyzed. SNR as a function of intrinsic noise intensity exhibits maximum, the maximum is the identifying characteristics of SR phenomenon. Increasing $q$ and $\tau_{d}$ are weaken SR, conversely, increasing $|A|$ enhances SR phenomenon in population system.

Next, to check the numerical simulations of the probability distributions of population levels and shift time to extinction the are presented, and are in agreement with the theoretical results. The numerical simulations in the probability distributions are consistent with the approximate theoretical results, which implies that the numerical simulations in population system with time delays and noises are credible.

In summary, time delay and noise widely exit in nature and often change fundamentally dynamics of the system. Our results shown that the time delay and noise cross-correlation intensity induce the structure of the probability density of population transfers from one state to the other. Moreover, we study the effects of the different time delay and noise cross-correlation intensity on the STE, NES and SR with a periodic signal. From the above findings, we can obtain further understanding of the roles of the time delay and cross-correlation intensity in this population model. As a result, we hope that these stimulate analysis could help understanding the state transitions in the population model. For the practical problems faced in real life, we will control the state of the dynamic system.

Acknowledgements. This work was supported by the National Natural Science Foundation of China (Granted Nos. 11975144 and 12002272).

\section{Author contributions}

CZ: writing - original draft preparation; TY: reviewing and editing, funding acquisition; S-XQ: reviewing and editing, funding acquisition. All the authors discussed the results and implications and commented on the manuscript at all stages. All the authors have read and approved the final manuscript.

Data Availability Statement This manuscript has no associated data or the data will not be deposited. [Authors' comment: The paper contents are purely theoretical, and did not need any data.]

\section{References}

1. M. Scheffer, S.R. Carpenter, T.M. Lenton, J. Bascompte, W. Brock, V. Dakos, J. Van de Koppel, I.A. Van de Leemput, S.A. Levin, E.H. Van Nes et al., Anticipating critical transitions. Science 338, 344-348 (2012)

2. C. Zeng, Q. Han, T. Yang, H. Wang, Z. Jia, Noise-and delay-induced regime shifts in an ecological system of vegetation. J. Stat. Mech. Theory Exp. 2013, P10017 (2013)

3. R. Mannella, P.V. McClintock, Noise in nonlinear dynamical systems. Contemp. Phys. 31, 179-194 (1990)

4. V.S. Anishchenko, V. Astakhov, A. Neiman, T. Vadivasova, L. Schimansky-Geier, Nonlinear dynamics of chaotic and stochastic systems: tutorial and modern developments (Springer, Berlin, 2007)

5. L. Gammaitoni, P. Hänggi, P. Jung, F. Marchesoni, Stochastic resonance. Rev. Mod. Phys. 70, 223 (1998)

6. M.D. McDonnell, N.G. Stocks, C.E.M. Pearce, D. Abbott, Stochastic resonance: from suprathreshold stochastic resonance to stochastic signal quantization. Cambridge University Press (2008)

7. W. Horsthemke, R. Lefever, Noise-induced nonequilibrium phase transitions. Noise Induced Trans. Theory Appl. Phys. Chem. Biol., 108-163 (1984)

8. P.S. Landa, P.V.E. McClintock, Changes in the dynamical behavior of nonlinear systems induced by noise. Phys. Rep. 323, 1-80 (2000) 
9. R.N. Mantegna, B. Spagnolo, Noise enhanced stability in an unstable system. Phys. Rev. Lett. 76, 563 (1996)

10. B. Spagnolo, A. Dubkov, N. Agudov, Enhancement of stability in randomly switching potential with metastable state. Eur. Phys. J. B Cond. Matter Complex Syst. 40, 273-281 (2004)

11. A. Fiasconaro, D. Valenti, B. Spagnolo, Role of the initial conditions on the enhancement of the escape time in static and fluctuating potentials. Phys. A Stat. Mech. Appl. 325, 136-143 (2003)

12. R.N. Mantegna, B. Spagnolo, Probability distribution of the residence times in periodically fluctuating metastable systems. Int. J. Bifurcat. Chaos 8, 783-790 (1998)

13. C. Guarcello, D. Valenti, A. Carollo, B. Spagnolo, Effects of lévy noise on the dynamics of sine-Gordon solitons in long Josephson junctions. J. Stat. Mech. Theory Exp. 2016, 054012 (2016)

14. L. Arnold, Trends and open problems in the theory of random dynamical systems, Probability towards, vol. 2000. (Springer, Berlin, 1998), pp. 34-46

15. R. Lefever, J.W. Turner, Sensitivity of a Hopf bifurcation to multiplicative colored noise. Phys. Rev. Lett. 56, 1631 (1986)

16. Y.-C. Lai, T. Tél, Transient chaos: complex dynamics on finite time scales, vol. 173 (Springer, Berlin, 2011)

17. Y. Luo, C. Zeng, Negative friction and mobilities induced by friction fluctuation. Chaos Interdiscipl. J. Nonlinear Sci. 30, 053115 (2020)

18. Y. Luo, C. Zeng, B.-Q. Ai, Strong-chaos-caused negative mobility in a periodic substrate potential. Phys. Rev. E 102, $042114(2020)$

19. A.S. Pikovsky, J. Kurths, Coherence resonance in a noise-driven excitable system. Phys. Rev. Lett. 78, 775 (1997)

20. B. Lindner, J. Garcia-Ojalvo, A. Neiman, L. Schimansky-Geier, Effects of noise in excitable systems. Phys. Rep. 392, 321-424 (2004)

21. F. Deng, Y. Luo, Y. Fang, F. Yang, C. Zeng, Temperature and friction-induced tunable current reversal, anomalous mobility and diffusions. Chaos Sol. Fract. 147, 110959 (2021)

22. M. Rietkerk, S.C. Dekker, P.C. De Ruiter, J. van de Koppel, Self-organized patchiness and catastrophic shifts in ecosystems. Science 305, 1926-1929 (2004)

23. L. Ridolfi, P. D'Odorico, F. Laio, Noise-Induced Phenomena in the Environmental Sciences (Cambridge University Press, Cambridge, 2011)

24. G. Denaro, D. Valenti, A. La Cognata, B. Spagnolo, A. Bonanno, G. Basilone, S. Mazzola, S. Zgozi, S. Aronica, C. Brunet, Spatio-temporal behaviour of the deep chlorophyll maximum in Mediterranean sea: development of a stochastic model for picophytoplankton dynamics. Ecol. Complex. 13, 21-34 (2013)

25. A. Giuffrida, D. Valenti, G. Ziino, B. Spagnolo, A. Panebianco, A stochastic interspecific competition model to predict the behaviour of listeria monocytogenes in the fermentation process of a traditional Sicilian salami. Eur. Food Res. Technol. 228, 767-775 (2009)

26. N. Pizzolato, A. Fiasconaro, D.P. Adorno, B. Spagnolo, Resonant activation in polymer translocation: new insights into the escape dynamics of molecules driven by an oscillating field. Phys. Biol. 7, 034001 (2010)
27. A. Mikhaylov, E. Gryaznov, A. Belov, D. Korolev, A. Sharapov, D. Guseinov, D. Tetelbaum, S. Tikhov, N. Malekhonova, A. Bobrov et al., Field-and irradiationinduced phenomena in memristive nanomaterials. Phys. Status Solidi (c) 13, 870-881 (2016)

28. C. Qiu, M. Al Kindi, A.S. Aladawi, I. Al Hatmi, A comprehensive study on microstructure and tensile behaviour of a selectively laser melted stainless steel. Sci. Rep. 8, 1-16 (2018)

29. A. Carollo, B. Spagnolo, D. Valenti, Uhlmann curvature in dissipative phase transitions. Sci. Rep. 8, 1-16 (2018)

30. Q. Liu, Y. Jia, Fluctuations-induced switch in the gene transcriptional regulatory system. Phys. Rev. E 70, 041907 (2004)

31. D. Zhang, H. Song, L. Yu, Q.-G. Wang, C. Ong, Setvalues filtering for discrete time-delay genetic regulatory networks with time-varying parameters. Nonlinear Dyn. 69, 693-703 (2012)

32. C. Wang, M. Yi, K. Yang, Time delay-accelerated transition of gene switch and-enhanced stochastic resonance in a bistable gene regulatory model. In: 2011 IEEE International Conference on Systems Biology (ISB), IEEE, pp. 101-110 (2011)

33. T. Yang, C. Zhang, Q. Han, C.-H. Zeng, H. Wang, D. Tian, F. Long, Noises-and delay-enhanced stability in a bistable dynamical system describing chemical reaction. Eur. Phys. J. B 87, 1-11 (2014)

34. P. Bressloff, S. Coombes, Traveling waves in a chain of pulse-coupled oscillators. Phys. Rev. Lett. 80, 4815 (1998)

35. D. Huber, L. Tsimring, Dynamics of an ensemble of noisy bistable elements with global time delayed coupling. Phys. Rev. Lett. 91, 260601 (2003)

36. T. Piwonski, J. Houlihan, T. Busch, G. Huyet, Delayinduced excitability. Phys. Rev. Lett. 95, 040601 (2005)

37. E. Craig, B. Long, J. Parrondo, H. Linke, Effect of time delay on feedback control of a flashing ratchet. EPL (Europhys. Lett.) 81, 10002 (2007)

38. Y. Wadop Ngouongo, M. Djolieu Funaye, G. Djuidjé Kenmoé, T. Kofané, Stochastic resonance in deformable potential with time-delayed feedback. Philos. Trans. R. Soc. A 379, 20200234 (2021)

39. N. Shao, J. Cheng, W. Chen The reproductive number $R_{0}$ of COVID-19 based on estimate of a statistical time delay dynamical system (2020). https://doi.org/ 10.1101/2020.02.17.20023747

40. K.Y. Ng, M.M. Gui, Covid-19: development of a robust mathematical model and simulation package with consideration for ageing population and time delay for control action and resusceptibility. Phys. D Nonlinear Phenomena 411, 132599 (2020)

41. S. Ghirlanda, M. Enquist, M. Perc, Sustainability of culture-driven population dynamics. Theor. Populat. Biol. 77, 181-188 (2010)

42. E. Bolhasani, Y. Azizi, D. Abdollahpour, J.M. Amjad, M. Perc, Control of dynamics via identical time-lagged stochastic inputs. Chaos Interdiscip. J. Nonlinear Sci. 30, $013143(2020)$

43. F. Nazarimehr, S. Jafari, M. Perc, J.C. Sprott, Critical slowing down indicators. EPL (Europhys. Lett.) 132, 18001 (2020) 
44. T. Frank, Delay Fokker-Planck equations, perturbation theory, and data analysis for nonlinear stochastic systems with time delays. Phys. Rev. E 71, 031106 (2005)

45. C. Zhang, L. Du, T. Wang, T. Yang, C. Zeng, C. Wang, Impact of time delay in a stochastic gene regulation network. Chaos Sol. Fract. 96, 120-129 (2017)

46. C. Yang, C. Zeng, B. Zheng, Prediction of regime shifts under spatial indicators in gene transcription regulation systems. EPL (Europhysics Letters) (2021)

47. C. Masoller, Noise-induced resonance in delayed feedback systems. Phys. Rev. Lett. 88, 034102 (2002)

48. T. Mori, S. Kai, Noise-induced entrainment and stochastic resonance in human brain waves. Phys. Rev. Lett. $\mathbf{8 8}, 218101(2002)$

49. A. Fiasconaro, A. Ochab-Marcinek, B. Spagnolo, E. Gudowska-Nowak, Monitoring noise-resonant effects in cancer growth influenced by external fluctuations and periodic treatment. Eur. Phys. J. B 65, 435-442 (2008)

50. A. La Cognata, D. Valenti, A. Dubkov, B. Spagnolo, Dynamics of two competing species in the presence of lévy noise sources. Phys. Rev. E 82, 011121 (2010)

51. D. Valenti, A. Fiasconaro, B. Spagnolo, Stochastic resonance and noise delayed extinction in a model of two competing species. Phys. A Stat. Mech. Appl. 331, 477$486(2004)$

52. B. Spagnolo, A. La Barbera, Role of the noise on the transient dynamics of an ecosystem of interacting species. Phys. A Stat. Mech. Appl. 315, 114-124 (2002)

53. A. Caruso, M. Gargano, D. Valenti, A. Fiasconaro, B. Spagnolo, Cyclic fluctuations, climatic changes and role of noise in planktonic foraminifera in the Mediterranean sea. Fluctuat. Noise Lett. 5, L349-L355 (2005)

54. R. Benzi, G. Parisi, A. Sutera, A. Vulpiani, Stochastic resonance in climatic change. Tellus 34, 10-16 (1982)

55. A. Patel, B. Kosko, Stochastic resonance in noisy spiking retinal and sensory neuron models. Neural Netw. 18, 467-478 (2005)

56. A.A. Zaikin, J. Kurths, L. Schimansky-Geier, Doubly stochastic resonance. Phys. Rev. Lett. 85, 227 (2000)

57. M. Rusconi, A. Zaikin, N. Marwan, J. Kurths, Effect of stochastic resonance on bone loss in osteopenic conditions. Phys. Rev. Lett. 100, 128101 (2008)

58. E. Volkov, E. Ullner, A. Zaikin, J. Kurths, Oscillatory amplification of stochastic resonance in excitable systems. Phys. Rev. E 68, 026214 (2003)

59. C. Zhou, J. Kurths, B. Hu, Array-enhanced coherence resonance: nontrivial effects of heterogeneity and spatial independence of noise. Phys. Rev. Lett. 87, 098101 (2001)

60. P.A. Stephens, W.J. Sutherland, Consequences of the Allee effect for behaviour, ecology and conservation. Trends Ecol. Evolut. 14, 401-405 (1999)

61. M. Khasin, E. Khain, L.M. Sander, Fast migration and emergent population dynamics. Phys. Rev. Lett. 109, $248102(2012)$

62. L. Arnold, W. Horsthemke, J. Stucki, The influence of external real and white noise on the LOTKAVOLTERRA model. Biomet. J. 21, 451-471 (1979)

63. A. Bahar, X. Mao, Stochastic delay Lotka-Volterra model. J. Math. Anal. Appl. 292, 364-380 (2004)

64. A.L. Pankratov, B. Spagnolo, Suppression of timing errors in short overdamped Josephson junctions. Phys. Rev. Lett. 93, 177001 (2004)
65. D. Valenti, G. Fazio, B. Spagnolo, Stabilizing effect of volatility in financial markets. Phys. Rev. E 97, 062307 (2018)

66. X. Mao, S. Sabanis, E. Renshaw, Asymptotic behaviour of the stochastic Lotka-Volterra model. J. Math. Anal. Appl. 287, 141-156 (2003)

67. L. Ramírez-Piscina, J.M. Sancho, A. HernándezMachado, Numerical algorithm for Ginzburg-Landau equations with multiplicative noise: application to domain growth. Phys. Rev. B 48, 125 (1993)

68. R.F. Fox, Functional-calculus approach to stochastic differential equations. Phys. Rev. A 33, 467 (1986)

69. C.W. Gardiner et al., Handbook of stochastic methods, vol. 3 (Springer, Berlin, 1985)

70. J. Hirsch, B. Huberman, D. Scalapino, Theory of intermittency. Phys. Rev. A 25, 519 (1982)

71. I. Dayan, M. Gitterman, G.H. Weiss, Stochastic resonance in transient dynamics. Phys. Rev. A 46, 757 (1992)

72. N. Agudov, B. Spagnolo, Noise-enhanced stability of periodically driven metastable states. Phys. Rev. E 64, $035102(2001)$

73. A.A. Dubkov, N.V. Agudov, B. Spagnolo, Noiseenhanced stability in fluctuating metastable states. Phys. Rev. E 69, 061103 (2004)

74. A. Fiasconaro, B. Spagnolo, S. Boccaletti, Signatures of noise-enhanced stability in metastable states. Phys. Rev. E 72, 061110 (2005)

75. D. Valenti, A. Carollo, B. Spagnolo, Stabilizing effect of driving and dissipation on quantum metastable states. Phys. Rev. A 97, 042109 (2018)

76. H.A. Kramers, Brownian motion in a field of force and the diffusion model of chemical reactions. Physica 7, 284-304 (1940)

77. W. Horsthemke, Non-equilibrium dynamics in chemical systems Noise induced transitions. (Springer, Berlin, 1984), pp. $150-160$

78. C. Van den Broeck, J. Parrondo, R. Toral, Noiseinduced nonequilibrium phase transition. Phys. Rev. Lett. 73, 3395 (1994)

79. J. García-Ojalvo, J. Sancho, Noise in spatially extended systems (Springer, Berlin, 2012) 